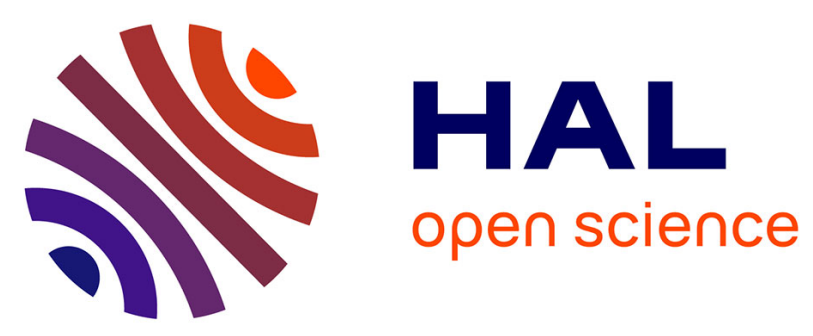

\title{
A new asymmetric synthesis of 2,6-cis-disubstituted-4-methylenpiperidines. Total synthesis of (+)-alkaloid 241D and (+)-isosolenopsin A
}

\author{
J. Monfray, Y. Gelas-Mialhe, J.-C. Gramain, R. Remuson
}

\section{- To cite this version:}

J. Monfray, Y. Gelas-Mialhe, J.-C. Gramain, R. Remuson. A new asymmetric synthesis of 2,6-cisdisubstituted-4-methylenpiperidines. Total synthesis of (+)-alkaloid 241D and (+)-isosolenopsin A. Tetrahedron: Asymmetry, 2005, 16, pp.1025-1034. 10.1016/j.tetasy.2005.01.018 . hal-00124913

\section{HAL Id: hal-00124913 https://hal.science/hal-00124913}

Submitted on 2 Mar 2007

HAL is a multi-disciplinary open access archive for the deposit and dissemination of scientific research documents, whether they are published or not. The documents may come from teaching and research institutions in France or abroad, or from public or private research centers.
L'archive ouverte pluridisciplinaire HAL, est destinée au dépôt et à la diffusion de documents scientifiques de niveau recherche, publiés ou non, émanant des établissements d'enseignement et de recherche français ou étrangers, des laboratoires publics ou privés. 


\title{
A new asymmetric synthesis of 2,6-cis-disubstituted 4-methylenepiperidines: total synthesis of (+)-alkaloid 241D and (+)-isosolenopsin $\mathrm{A}$
}

\author{
Jérémy Monfray, Yvonne Gelas-Mialhe, Jean-Claude Gramain and Roland Remuson* \\ UMR 6504, CNRS, Université Blaise Pascal (Clermont-Ferrand), 63177 Aubière cedex, France
}

Received 2 December 2004; accepted 6 January 2005

\begin{abstract}
A highly diastereoselective synthesis of 2,6-cis-disubstituted-4-methylenepiperidines based on a Mannich type intramolecular cyclization of an allylsilane on an iminium ion is described. The synthetic potential of this methodology is demonstrated by the enantioselective synthesis of two natural piperidine alkaloids: (+)-alkaloid 241D and (+)-isosolenopsin A.
\end{abstract}

(c) 2005 Elsevier Ltd. All rights reserved.

\section{Introduction}

Many natural biologically active compounds contain the piperidine ring system as a common structural element. Among the numerous piperidines, cis- and trans-2,6dialkylpiperidines represent an important class of alkaloids isolated from insects, amphibians or plants. ${ }^{1}$ For instance, dihydropinidine 1 was found in the Mexican beetle Epilachna varivestis. ${ }^{2}$ Solenopsins $\mathbf{2}$ and isosolenopsins $\mathbf{3}$ are extracted from the fire ants' venom of the genus Solenopsis, ${ }^{3}$ while alkaloid 241D 4 was isolated from the poison frog Dendrobates ${ }^{4}$ (Fig. 1).

The stereoselective synthesis of piperidines, and notably 2,6-cis-disubstituted piperidines, has received considerable attention ${ }^{5,6}$ due to the broad range of their biological activity. ${ }^{7}$ As part of our programme to expand the synthetic utility of allylsilyl-functionalized substrates for the synthesis of natural products, ${ }^{8}$ we have applied

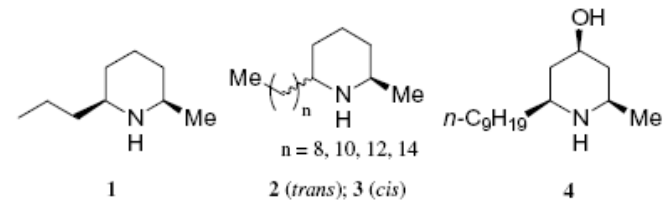

Figure 1. Examples of natural piperidines.

\footnotetext{
${ }^{*}$ Corresponding author. Tel.: +33(0)4 734076 45; fax: +33(0)4 7340 77 17; e-mail: remuson@chimie.univ-bpclermont.fr
}

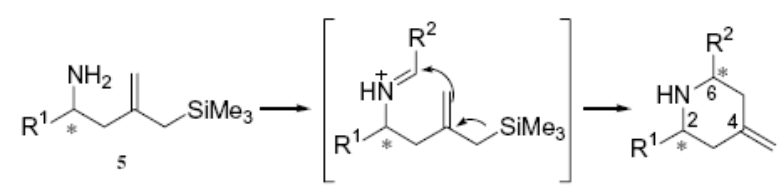

Scheme 1. Formation of the piperidine ring by intramolecular addition of an allylsilane on an iminium ion.

this strategy to the synthesis of these compounds. In this case, the piperidine ring would be formed by a Mannich type intramolecular cyclization reaction starting from substituted aminoallylsilanes 5 (Scheme 1).

\section{Results and discussion}

Previously, ${ }^{9}$ we have described the enantioselective synthesis of substrates $\mathbf{5}$. They were prepared from $\alpha, \beta$-ethylenic esters 6 in four steps in 21-67\% overall yields and $80-84 \%$ enantiomeric excesses (Scheme 2).

\subsection{Synthesis of 2,6-disubstituted-4-methylenepiperidines}

Since numerous natural piperidines are substituted by a methyl group at the 2 position, we have chosen to prepare piperidines $17-22$ by condensation of methyl substituted aminoallylsilane $(R)-5$ a on carbonyl compounds 11-16 (Scheme 3).

Reaction of aminoallylsilane $(R)-5$ a $(e e=82 \%)$ with aldehydes 11-15 in the presence of trifluoroacetic acid 


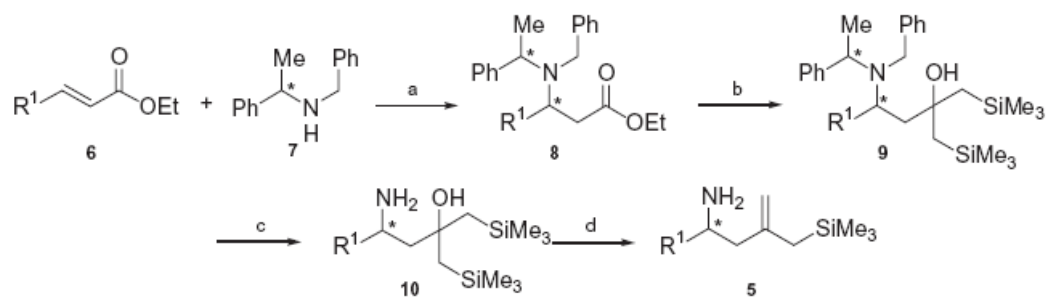

Scheme 2. Reagents and conditions: (a) $n$-BuLi, THF, $0{ }^{\circ} \mathrm{C}$; (b) $\mathrm{Me}_{3} \mathrm{SiMgCl}_{\mathrm{M}}$ then $\mathrm{CeCl}_{3}, \mathrm{THF}$, rt, 3 days; (c) $\mathrm{Pd}(\mathrm{OH})_{2}, \mathrm{H}_{2}, \mathrm{MeOH}, \mathrm{H}{ }_{2} \mathrm{O}, \mathrm{THF}$, AcOH, 24 h; (d) $\mathrm{HCl} 1 \mathrm{M}$, diethyl ether, 1-24 h.

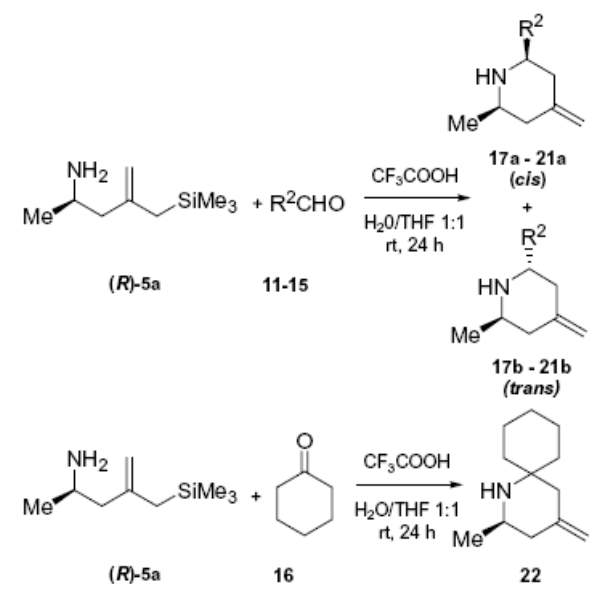

Scheme 3. Synthesis of 4-methylenepiperidines from aminoallylsilane (R)-5a.

in a mixture of water-THF (1:1) at room temperature for $24 \mathrm{~h}$ led to a mixture of cis- and trans-4-methylenepiperidines. The results are summarized in Table 1 . In all cases, the cis-diastereomers were predominant. The diastereoisomeric excesses, which were found to be better than $82 \%$, were determined by ${ }^{1} \mathrm{H}$ NMR spectroscopy on the signals corresponding to the methylene protons. The relative configuration of the cis-diastereoisomers 17a-21a was established unambiguously by ${ }^{1} \mathrm{H}$ NMR spectroscopy. For instance, the ${ }^{1} \mathrm{H}$ NMR spectrum of 21a showed triplets for $\mathrm{H}-3_{\mathrm{ax}}$ and $\mathrm{H}-5_{\mathrm{ax}}$ with $J=12.8 \mathrm{~Hz}$ and $J=12.9 \mathrm{~Hz}$ corresponding, respectively, to geminal and trans-diaxial couplings indicative of a cis-stereochemistry for the 2,6-disubstituted piperidine ring. These results were confirmed by NOE experiments on isomers 21a and 21b (Fig. 2).

To explain such a diastereoselectivity, we considered the transition states $\mathbf{A}$ and $\mathbf{B}$ (Scheme 4). It appears that the transition state $\mathbf{B}$ leading to the 2,6-trans isomer is disfavoured due to a 1,3-diaxial interaction compared to the transition state A leading to the 2,6-cis isomer.

The enantiomeric purity of piperidines 18a-21a and $\mathbf{2 2}$ was determined using two different techniques: GCMS with Mosher's acid derivatives and ${ }^{1} \mathrm{H}$ NMR with $(R)$-mandelic acid derivatives. The results (Table 1) have shown that a partial racemization occurred during the cyclization step. This racemization can be explained by an aza-Cope type rearrangement. It has already been observed previously by other authors ${ }^{10}$ during the addition of vinyl and allylsilanes on iminium salts.

In order to improve yields and minimize racemization during the cyclization step, we studied the preparation of piperidines 17-22 from $\beta$-aminohydroxysilanes 10, precursors of $\beta$-aminoallylsilanes 5 (Scheme 5).

Reaction of $\beta$-aminohydroxysilanes $\mathbf{1 0}$ with carbonyl derivatives 11, 13-16 and 23 in the presence of trifluoroacetic acid (10 equiv) in a mixture of water-THF (1:1) at room temperature for 3 days led to a mixture of cis- and trans-4-methylenepiperidines. The results are summarized in Table 2.

As in the preceding results, in all cases, the cis-diastereoisomers were predominant. Comparison of these results with those mentioned in Table 1 shows that diastereoselectivity is about the same but enantioselectivity is significantly increased when the piperidine ring is achieved from aminohydroxysilanes.

A mechanism that could account for the formation of 4methylenepiperidines from $\beta$-aminohydroxysilanes $\mathbf{1 0}$ is depicted in Scheme 6. Cyclization might proceed by the following sequence: after formation of 1,3-oxazinane $\mathbf{2 4}$ and its protonation, iminium ion $\mathbf{2 5}$ is generated. Protonation of the oxygen atom leads to allylsilane $\mathbf{2 6}$ and finally cyclization affords the expected piperidine. In this case, the allylsilane moiety is generated in situ and reacts instantaneously with the iminium ion previously formed.

The intermediate 1,3-oxazinanes $\mathbf{2 4}$ were unambiguously displayed by isolation of $24 a\left(\mathrm{R}^{1}=\mathrm{Me}, \mathrm{R}^{2}=n\right.$ $\left.\mathrm{C}_{9} \mathrm{H}_{19}\right)$ and $24 \mathbf{b}\left(\mathrm{R}^{1}=\mathrm{Me}, \mathrm{R}^{2}=\mathrm{Ph}\right)$ from, respectively, condensation of decanal 13 and benzaldehyde 15 with $\beta$-aminohydroxysilane $(R)$-10a without adding trifluoroacetic acid. Furthermore, treatment of 24a with trifluoroacetic acid led to piperidine $\mathbf{1 9} \mathbf{a}$.

\subsection{Total synthesis of piperidine alkaloids}

The preceding results have shown that the best way to access to 4-methylenepiperidines is from $\beta$-aminohydroxysilanes 10. We have used this strategy for the total synthesis of piperidine alkaloids (+)-alkaloid 241D 4 and (+)-isosolenopsin A 3a.

2.2.1. Synthesis of (+)-alkaloid 241D 4. Racemic alkaloid $241 \mathrm{D}$ was shown to have interesting biological activ- 
Table 1. Preparation of 4-methylenepiperidines from aminoallylsilane 5a

\begin{tabular}{|c|c|c|c|c|}
\hline Carbonyl compound & Major product & $\mathrm{De}^{\mathrm{a}}(\%)$ & Yield $^{\mathrm{b}}(\%)$ & $\mathrm{Ee}^{\mathrm{b}}(\%)$ \\
\hline $\begin{array}{c}n-\mathrm{C}_{3} \mathrm{H}_{7} \mathrm{CHO} \\
11\end{array}$ & $17 \mathbf{a}$ & 84 & 49 & n.d. $\left([\alpha]_{D}^{21}=-4\right)$ \\
\hline $\begin{array}{c}\mathrm{CH}_{3} \mathrm{CH}=\mathrm{CHCHO} \\
12\end{array}$ & 18a & n.d. & 32 & $78^{\mathrm{c}}$ \\
\hline $\begin{array}{c}n-\mathrm{C}_{9} \mathrm{H}_{19} \mathrm{CHO} \\
13\end{array}$ & 19a & 84 & 49 & $28^{\mathrm{d}}$ \\
\hline $\begin{array}{c}n-\mathrm{C}_{11} \mathrm{H}_{23} \mathrm{CHO} \\
\mathbf{1 4}\end{array}$ & (11 & 82 & 58 & $38^{\mathrm{d}}$ \\
\hline $\begin{array}{c}\mathrm{PhCHO} \\
15\end{array}$ & $21 \mathrm{a}$ & 86 & 70 & $76^{\mathrm{d}}$ \\
\hline 16 & 22 & 86 & 46 & $24^{\mathrm{c}}$ \\
\hline
\end{tabular}

${ }^{\mathrm{a}}$ Determined by ${ }^{1} \mathrm{H}$ NMR on the crude product.

${ }^{\mathrm{b}}$ Determined on the major isolated piperidine.

${ }^{\mathrm{C}}$ Determined by ${ }^{1} \mathrm{H}$ NMR of the $(R)$-mandelic acid ammonium salt.

${ }^{\mathrm{d}}$ Determined by GC-MS of the Mosher's acid derivative.

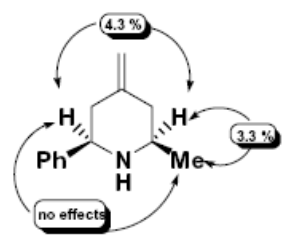

21a (cis)

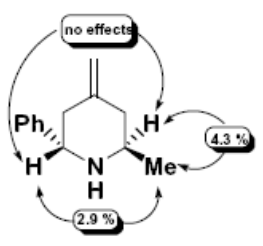

21b (trans)

Figure 2. NOE effects on cis- and trans-piperidines 21.

ities: for example, it stops the action of acetylcholine by a noncompetitive blocker of the nicotinic receptor channel

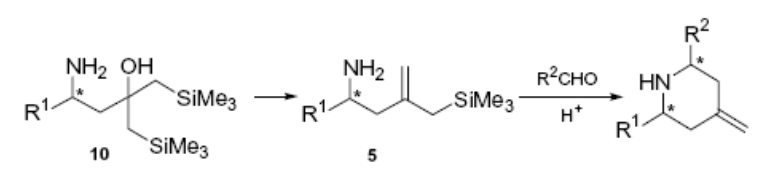

Scheme 5. Synthesis of 4-methylenepiperidines from $\beta$-aminohydroxyallylsilanes $\mathbf{1 0 .}$

complex. ${ }^{11}$ Also it is a potent inhibitor of binding of $\left[{ }^{3} \mathrm{H}\right]$ perhydrohistrionicotoxin to nicotinic receptor channels of electroplax membranes. ${ }^{12}$ Various asymmetric synthesis of $(+)$-alkaloid 241D have been described. ${ }^{13}$ We have used methylenepiperidine 19a to prepare this alkaloid

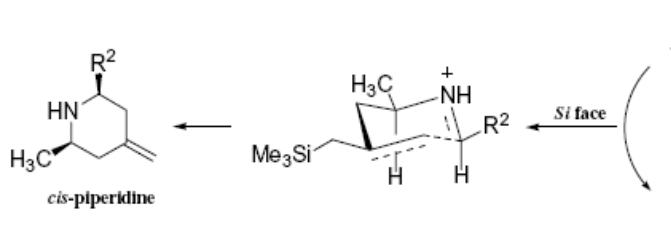

A<smiles>[R2]C=N[C@@H](C)CC(=C)C[SiH3]C</smiles>

B

Scheme 4. Explanation of diastereoselectivity. 
Table 2. Preparation of 4-methylenepiperidines from $\beta$-aminohydroxysilanes $\mathbf{1 0}$

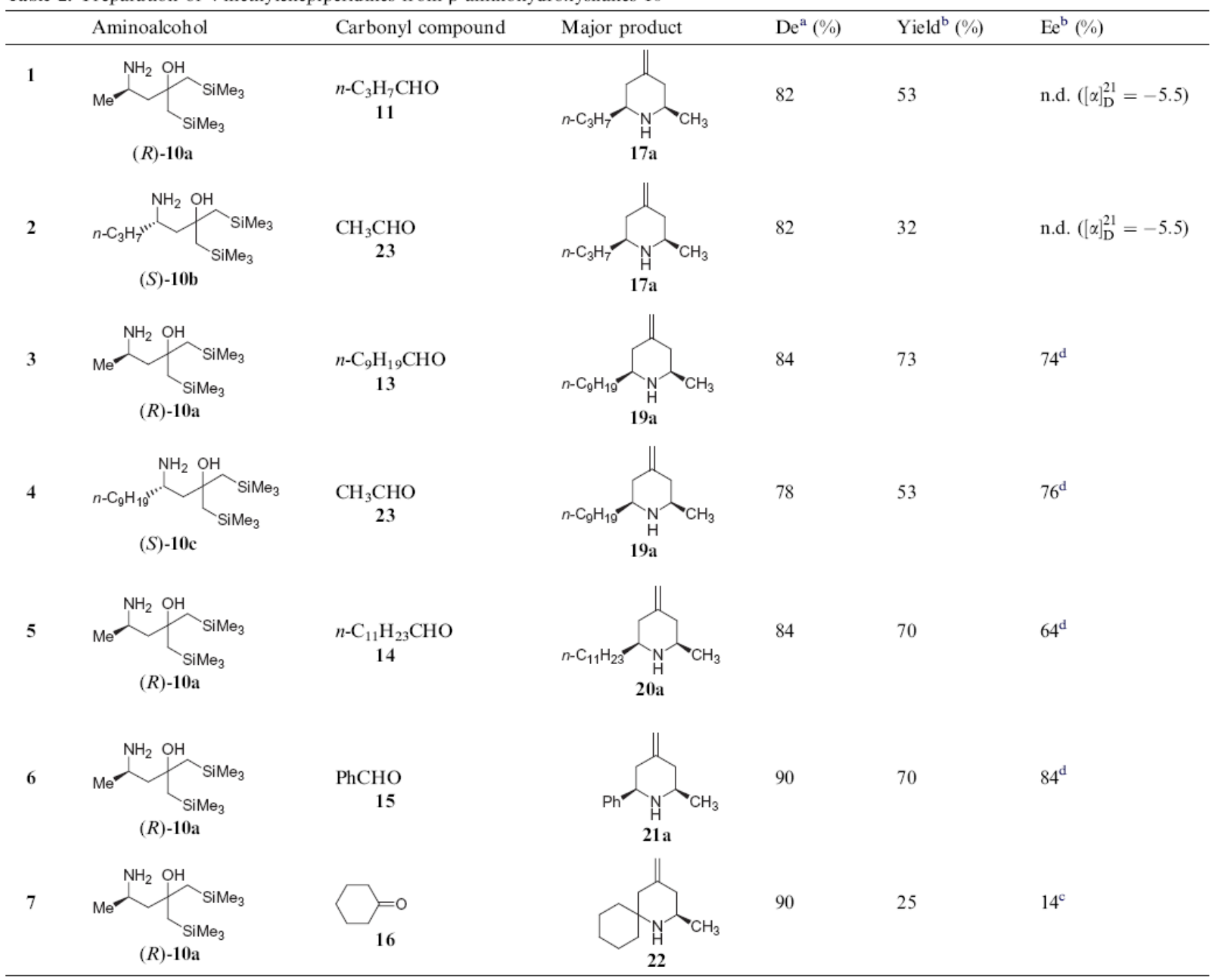

${ }^{\text {a }}$ Determined by ${ }^{1} \mathrm{H}$ NMR on the crude product.

${ }^{\mathrm{b}}$ Determined on the major isolated piperidine.

${ }^{\mathrm{c}}$ Determined by ${ }^{1} \mathrm{H}$ NMR of the $(R)$-mandelic acid ammonium salt.

${ }^{\mathrm{d}}$ Determined by GC-MS of the Mosher's acid derivative.

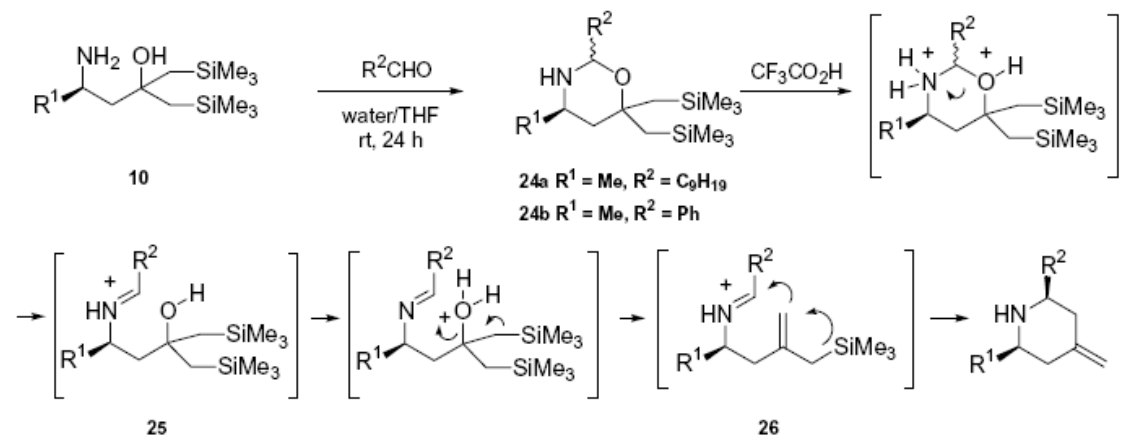

Scheme 6. Mechanism of the formation of 4-methylenepiperidines from aminohydroxysilanes $\mathbf{1 0 .}$

according to the sequence outlined in Scheme 7. Oxidation of 4-methylenepiperidine 19a with osmium tetroxide in the presence of $\mathrm{Na}_{3} \mathrm{H}_{3} \mathrm{IO}_{6}$ in acetic acid led to piperidin-4-one 25 in $68 \%$ yield. The stereoselective reduction 


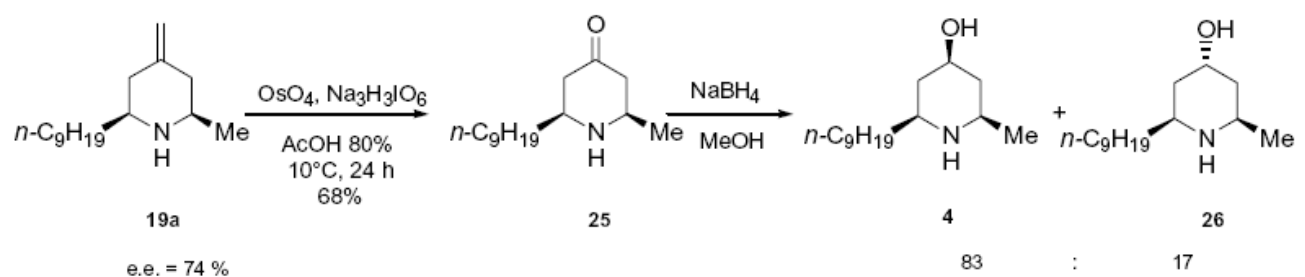

Scheme 7. Synthesis of (+)-alkaloid 241D 4.

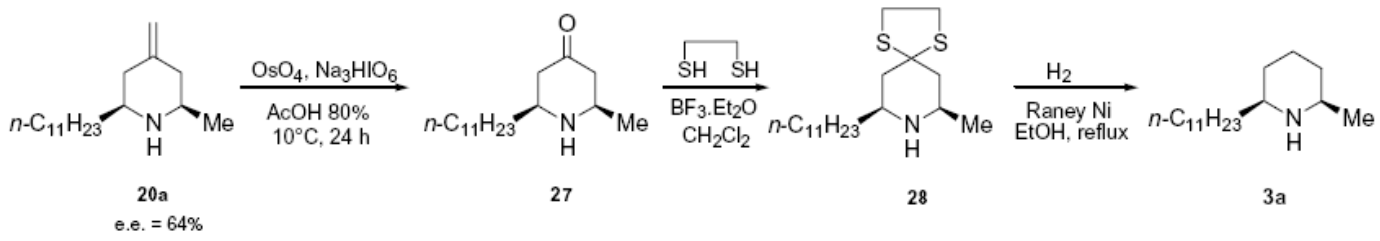

Scheme 8. Synthesis of the (+)-isosolenopsin A hydrochloride 3a.

of 25 with sodium borohydride afforded (+)-alkaloid $241 \mathrm{D} 4$ and its C-4 epimer 26 in a ratio of 83:17. The natural product was isolated in a $66 \%$ yield. Consequently, $(+)$-alkaloid 241D was obtained in six steps from methyl crotonate in an overall yield of $23 \%$ and an enantiomeric excess of $74 \%\left\{[\alpha]_{\mathrm{D}}^{25}=+5.5(c \quad 1.04, \mathrm{MeOH})\right.$, lit. ${ }^{13 \mathrm{a}}$ $\left.[\alpha]_{\mathrm{D}}^{25}=+6.5(c 2, \mathrm{MeOH})\right\}$.

2.2.2. Synthesis of (+)-isosolenopsin A 3a. (+)-Isosolenopsin $\mathrm{A}$ has been shown to have numerous biological activities such as antibacterian, antifongic, insecticide and phytocid. According to a similar sequence, (+)-isosolenopsin $\mathrm{A}^{14}$ was prepared from 4-methylenepiperidine 20a. (Scheme 8). Oxidation of 20a with osmium tetroxide and $\mathrm{Na}_{3} \mathrm{H}_{3} \mathrm{IO}_{6}$ led to piperidin-4-one 27 in $67 \%$ yield. Treatment of 27 with an excess of ethane dithiol in the presence of $\mathrm{BF}_{3} \cdot \mathrm{Et}_{2} \mathrm{O}$ gave the dithiolane derivative $\mathbf{2 8}$ in $82 \%$ yield. Finally, $\mathbf{2 8}$ was converted into (+)isosolenopsin A 3a (isolated as its hydrochloride salt) in $43 \%$ yield using Raney nickel in reflux ethanol.

$(+)$-Isosolenopsin $\mathrm{A}(\mathrm{HCl})$ was obtained in seven steps from ethyl crotonate in $11 \%$ overall yield and $64 \%$ enantiomeric excess. $\left\{[\alpha]_{\mathrm{D}}^{25}=+5.5\left(c 1.00, \mathrm{CHCl}_{3}\right)\right.$, lit. ${ }^{14 \mathrm{c}}$ $\left.[\alpha]_{\mathrm{D}}=+10.0\left(c 1.17, \mathrm{CHCl}_{3}\right)\right\}$.

\section{Conclusion}

We have described a new approach for the enantioselective synthesis of 2,6-cis-disubstituted-4-methylenepiperidines. The key step of the sequence is an intramolecular allylsilane-iminium cyclization. Piperidines were obtained with good yields and excellent diastereoselectivity. Moderate enantiomeric excesses were measured when a $\beta$-aminoallylsilane was used as starting material, a better enantioselectivity was observed when we started from $\beta$-aminohydroxysilanes. This methodology was applied to the synthesis of (+)-alkaloid 241D and (+)isosolenopsin $\mathrm{A}$.

\section{Experimental}

\subsection{General}

Commercially available materials were used without further purification. THF used for moisture sensitive operations were distilled from potassium/benzophenone under an argon atmosphere. All moisture sensitive reactions were carried out in flame-dried glassware under an argon atmosphere. Analytical thin layer chromatography (TLC) was performed on Merck silica gel $60 \mathrm{~F}_{254}$ plates. Visualization on TLC was achieved by use of UV light $(254 \mathrm{~nm})$, iodide, ninhydrin or vanillin followed by heating. Flash chromatography was performed using Merck silica gel 40-60 $\mu \mathrm{m}$.

Infrared spectra were recorded on a Perkin-Elmer 881 (spectra in solution) or on a Perkin-Elmer FTIR Spectrometer Paragon 500 (film). Only selected absorbances are reported. Optical rotations were measured on a Jasco DIP-370 polarimeter at $589 \mathrm{~nm}$ (Na D-line).

Nuclear magnetic resonance (NMR) spectra were recorded on a Bruker Avance 400 spectrometer at operating frequencies of $400 \mathrm{MHz}\left({ }^{1} \mathrm{H}\right.$ NMR) or $100 \mathrm{MHz}\left({ }^{13} \mathrm{C}\right.$ NMR). Chemical shifts $(\delta)$ are given in ppm relative to $\mathrm{CDCl}_{3}\left(\delta=7.27 \mathrm{ppm}\right.$ for ${ }^{1} \mathrm{H}, \delta=77.1 \mathrm{ppm}$ for $\left.{ }^{13} \mathrm{C}\right)$ and coupling constants $(J)$ in hertz. Multiplicity is tabulated as $\mathrm{s}$ for singlet, $\mathrm{d}$ for doublet, $\mathrm{t}$ for triplet, $\mathrm{q}$ for quadruplet, $\mathrm{m}$ for multiplet; the prefix br is used for a broad signal.

Mass spectra (MS) in electronic ionization mode (EI) were recorded on an Agilent $6890 \mathrm{~N}$ mass spectrometer (GC/MS). Other spectra were performed on a Hewlett-Packard 5989B spectrometer. High-resolution mass spectra (HRMS) were recorded at the Centre Régional de Mesures Physique de l'Ouest (CRMPO, University of Rennes I, France) on a Varian Mat 311 spectrometer (EI) or on a Micromass ZABSpecTOF (ESI). 
Microanalysis were carried out at the Laboratoire Central de Microanalyse du CNRS (Vernaison, France).

\subsection{General procedure for preparation of 8}

To a solution of $(R)$ - or $(S)$ - $N$-benzyl- $N^{\prime}-\alpha$-methylbenzylamine 7 (1.1 equiv, $0.25-0.32 \mathrm{~mol} \mathrm{~L}^{-1}$ ) in dry THF was added dropwise at $0^{\circ} \mathrm{C}$ a solution of $n$-butyllithium (1.2 equiv) in hexane $\left(c=1.6 \mathrm{~mol} \mathrm{~L}^{-1}\right)$. After stirring for $15 \mathrm{~min}$, a solution of the $\alpha, \beta$-ethylenic ester 6 (23-35 mmol, $1.1-1.6 \mathrm{~mol} \mathrm{~L}^{-1}$ ) in dry THF was added at $0^{\circ} \mathrm{C}$. After stirring for $1 \mathrm{~h}$, the mixture was hydrolyzed with a saturated solution of ammonium chloride. The layers were separated and the aqueous layer extracted with diethyl ether. The combined organic layers were dried over $\mathrm{MgSO}_{4}$ and concentrated. The crude product was purified by flash chromatography (cyclohexane/AcOEt 95:5). A mixture of two diastereoisomers was obtained.

4.2.1. Ethyl $\left(3 R, 3^{\prime} R\right)-3-\left(N\right.$ - $\alpha$-methylbenzyl- $N^{\prime}$-benzylamino)-butanoate 8a. Pale yellow oil; yield: $83 \%$; TLC: $R_{\mathrm{f}}=0.37$ (cyclohexane/AcOEt, 95:5); de $=82 \%$; $[\alpha]_{\mathrm{D}}^{25}=+3\left(c 1.12, \mathrm{CHCl}_{3}\right)$; IR $\left(\mathrm{CCl}_{4}\right): v\left(\mathrm{~cm}^{-1}\right) 1730$; ${ }^{1} \mathrm{H}$ NMR $\delta$ major diastereoisomer $1.20(\mathrm{~d}, 3 \mathrm{H}$, $J=6.7 \mathrm{~Hz}), 1.22(\mathrm{t}, 3 \mathrm{H}, J=7.1 \mathrm{~Hz}), 1.41(\mathrm{~d}, 3 \mathrm{H}$, $J=6.9 \mathrm{~Hz}), 2.29$ (AB part of $\mathrm{ABX}$ system, $2 \mathrm{H}$, $\Delta v=100 \mathrm{~Hz}), \quad \delta_{\mathrm{A}}=2.42 \quad\left(\mathrm{dd}, \quad 1 \mathrm{H}, \quad J_{\mathrm{AB}}=14.1 \mathrm{~Hz}\right.$, $J_{\mathrm{AX}}=6.0 \mathrm{~Hz}, \quad \delta_{\mathrm{B}}=2.17 \quad\left(\mathrm{dd}, \quad 1 \mathrm{H}, \quad J_{\mathrm{AB}}=14.1 \mathrm{~Hz}\right.$, $\left.J_{\mathrm{BX}}=8.0 \mathrm{~Hz}\right)$ ), 3.51 (X part of $\mathrm{ABX}$ system, m, $1 \mathrm{H}$, $\left.J_{\mathrm{AX}}=6.0 \mathrm{~Hz}, J_{\mathrm{BX}}=8.0 \mathrm{~Hz}\right), 3.77(\mathrm{AB}$ system, 2H, $\Delta v=20 \mathrm{~Hz}, \delta_{\mathrm{A}}=3.79\left(\mathrm{~d}, 1 \mathrm{H}, J_{\mathrm{AB}}=14.7 \mathrm{~Hz}\right), \delta_{\mathrm{B}}=3.75$ $\left.\left(\mathrm{d}, 1 \mathrm{H}, J_{\mathrm{AB}}=14.7 \mathrm{~Hz}\right)\right), 3.92-4.11(\mathrm{~m}, 3 \mathrm{H}), 7.24-7.49$ $(\mathrm{m}, 10 \mathrm{H}) ;{ }^{13} \mathrm{C}$ NMR $\delta$ major diastereoisomer 14.2 , 18.0, $18.6\left(\mathrm{CH}_{3}\right), 39.9,49.7\left(\mathrm{CH}_{2}\right), 50.1,57.9(\mathrm{CH}), 60.2$ $\left(\mathrm{CH}_{2}\right), 126.6,126.7,127.8,128.0,128.1,128.2,128.4$ $(\mathrm{CH}), 141.7,144.3,172.5(\mathrm{C})$; MS for $\mathrm{C}_{21} \mathrm{H}_{27} \mathrm{NO}_{2}: \mathrm{m} / z$ 325. Anal. Calcd for $\mathrm{C}_{21} \mathrm{H}_{27} \mathrm{NO}_{2}$ : C, 77.50; H, 8.36; N, 4.30. Found: $\mathrm{C}, 77.92 ; \mathrm{H}, 8.45 ; \mathrm{N}, 4.07$.

4.2.2. Ethyl $\left(3 S, 3^{\prime} S\right)-3-\left(N-\alpha\right.$-methylbenzyl- $N^{\prime}$-benzylamino)-hexanoate 8b. Yellow oil; yield: $70 \%$; TLC: $R_{\mathrm{f}}=0.33 \quad$ (cyclohexane/AcOEt, $95: 5$ ); $\quad \mathrm{de}=82 \%$; $[\alpha]_{\mathrm{D}}^{25}=-20\left(c 1.31, \mathrm{CHCl}_{3}\right)$; IR $\left(\mathrm{CCl}_{4}\right): v\left(\mathrm{~cm}^{-1}\right) 1735$; ${ }^{1} \mathrm{H}$ NMR $\delta$ major diastereoisomer $0.78(\mathrm{t}, 3 \mathrm{H}$, $J=7.2 \mathrm{~Hz}), 1.08(\mathrm{t}, 3 \mathrm{H}, J=7.2 \mathrm{~Hz}), 1.12-1.22(\mathrm{~m}, 2 \mathrm{H})$, $1.25(\mathrm{~d}, 3 \mathrm{H}, J=6.8 \mathrm{~Hz}), 1.36-1.57(\mathrm{~m}, 2 \mathrm{H}), 1.93$ (AB part of ABX system, $2 \mathrm{H}, \Delta v=20 \mathrm{~Hz}, \delta_{\mathrm{A}}=1.95(\mathrm{dd}, 1 \mathrm{H}$, $\left.J_{\mathrm{AB}}=14.5 \mathrm{~Hz}, \quad J_{\mathrm{AX}}=4.2 \mathrm{~Hz}\right), \quad \delta_{\mathrm{B}}=1.90 \quad(\mathrm{dd}, 1 \mathrm{H}$, $\left.\left.J_{\mathrm{AB}}=14.6 \mathrm{~Hz}, J_{\mathrm{BX}}=8.9 \mathrm{~Hz}\right)\right), 3.24(\mathrm{X}$ part of ABX system, m, $1 \mathrm{H}, J_{\mathrm{AX}}=4.2 \mathrm{~Hz}, J_{\mathrm{BX}}=8.9 \mathrm{~Hz}$ ), 3.57 (AB system, $2 \mathrm{H}, \Delta v=99 \mathrm{~Hz}, \delta_{\mathrm{A}}=3.70\left(\mathrm{~d}, 1 \mathrm{H}, J_{\mathrm{AB}}=14.9 \mathrm{~Hz}\right)$, $\left.\delta_{\mathrm{B}}=3.45\left(\mathrm{~d}, 1 \mathrm{H}, \quad J_{\mathrm{AB}}=14.9 \mathrm{~Hz}\right)\right), 3.74(\mathrm{q}, 1 \mathrm{H}$, $J=7.0 \mathrm{~Hz}), 3.85-3.99(\mathrm{~m}, 2 \mathrm{H}), 7.10-7.35(\mathrm{~m}, 10 \mathrm{H}) ;{ }^{13} \mathrm{C}$ NMR $\delta$ major diastereoisomer 14.2, 14.3, $19.8\left(\mathrm{CH}_{3}\right)$, $20.3,35.9,36.9,50.0\left(\mathrm{CH}_{2}\right), 53.8,58.0(\mathrm{CH}), 60.1$ $\left(\mathrm{CH}_{2}\right), 126.6,126.8,126.9,127,128.0,128.1,128.2$, $128.3(\mathrm{CH}), \quad 143.2, \quad 141.9, \quad 173.0 \quad(\mathrm{C}) ; \mathrm{MS}$ for $\mathrm{C}_{23} \mathrm{H}_{31} \mathrm{NO}_{2}: m / z$ 353. Anal. Calcd for $\mathrm{C}_{23} \mathrm{H}_{31} \mathrm{NO}_{2}: \mathrm{C}$, 78.15; H, 8.84; N, 3.96. Found: C, 78.10; H, 8.65; N, 3.87.

4.2.3. Ethyl $\left(3 S, 3^{\prime} S\right)-3-\left(N\right.$-methylbenzyl- $N^{\prime}$-benzylamino)-dodecanoate 8c. Yellow oil; yield: 69\%; TLC: $R_{\mathrm{f}}=0.48 \quad$ (cyclohexane/AcOEt, $95: 5$ ); $\quad \mathrm{de}=80 \%$;
$[\alpha]_{\mathrm{D}}^{25}=-11\left(c 1.10, \mathrm{CHCl}_{3}\right) ;$ IR $\left(\mathrm{CCl}_{4}\right): \quad v \quad\left(\mathrm{~cm}^{-1}\right)$ $1722 ;{ }^{1} \mathrm{H}$ NMR $\delta$ major diastereoisomer $0.95(\mathrm{t}, 3 \mathrm{H}$, $J=6.9 \mathrm{~Hz}), 1.23(\mathrm{t}, 3 \mathrm{H}, J=7.2 \mathrm{~Hz}), 1.25-1.36(\mathrm{~m}$, $14 \mathrm{H}), 1.39(\mathrm{~d}, 3 \mathrm{H}, J=6.8 \mathrm{~Hz}), 1.51-1.65(\mathrm{~m}, 1 \mathrm{H}, \mathrm{H}-$ 4), 2.08 (AB part of ABX system, $2 \mathrm{H}, \mathrm{H}-2, \Delta v=21 \mathrm{~Hz}$, $\delta_{\mathrm{A}}=2.10\left(\mathrm{dd}, 1 \mathrm{H}, \quad J_{\mathrm{AB}}=14.4 \mathrm{~Hz}, J_{\mathrm{AX}}=4.1 \mathrm{~Hz}\right)$, $\left.\delta_{\mathrm{B}}=2.05\left(\mathrm{dd}, 1 \mathrm{H}, J_{\mathrm{AB}}=14.4 \mathrm{~Hz}, J_{\mathrm{BX}}=8.7 \mathrm{~Hz}\right)\right), 3.36$ $(\mathrm{m}, 1 \mathrm{H}), 3.72\left(\mathrm{AB}\right.$ system, $2 \mathrm{H}, \Delta v=99 \mathrm{~Hz}, \delta_{\mathrm{A}}=3.84$ (d, $\left.1 \mathrm{H}, \quad J_{\mathrm{AB}}=15.0 \mathrm{~Hz}\right), \quad \delta_{\mathrm{B}}=3.60 \quad(\mathrm{~d}, \quad 1 \mathrm{H}$, $\left.\left.J_{\mathrm{AB}}=15.0 \mathrm{~Hz}\right)\right), 3.89(\mathrm{q}, 1 \mathrm{H}, J=7.0 \mathrm{~Hz}), 4.0-4.13(\mathrm{~m}$, $2 \mathrm{H}), 7.25-7.50(\mathrm{~m}, 10 \mathrm{H}) ;{ }^{13} \mathrm{C}$ NMR $\delta$ major diastereoisomer 14.3, $19.7\left(\mathrm{CH}_{3}\right), 22.7,27.0,29.5,29.6,32.0$, 33.6, 36.9, $50.0\left(\mathrm{CH}_{2}\right), 54.1,56.9(\mathrm{CH}), 60.1\left(\mathrm{CH}_{2}\right)$, $126.8,126.9,128.1,128.7(\mathrm{CH}), 141.8,143.3,172.9$ (C); MS for $\mathrm{C}_{29} \mathrm{H}_{43} \mathrm{NO}_{2}: m / z$ 465. Anal. Calcd for $\mathrm{C}_{29} \mathrm{H}_{43} \mathrm{NO}_{2}$ : C, 79.59; H, 9.90; N, 3.20. Found: C, $79.76 ; \mathrm{H}, 10.13 ; \mathrm{N}, 3.10$.

\subsection{General procedure for the preparation of 9}

Powdered $\mathrm{CeCl}_{3} \cdot 7 \mathrm{H}_{2} \mathrm{O}$ (4.5-4.6 equiv) was dried under vacuum $(0.5 \mathrm{mmHg})$ for 3 days at $120-130{ }^{\circ} \mathrm{C}$ while stirring. The flask was flushed with argon, then dry THF ( $7 \mathrm{~mL} / \mathrm{g}$ of $\mathrm{CeCl}_{3} \cdot 7 \mathrm{H}_{2} \mathrm{O}$ ) added. The white suspension was stirred at room temperature for an additional $2 \mathrm{~h}$. This slurry was cooled to $-78^{\circ} \mathrm{C}$ and trimethylsilyl methylmagnesium chloride (4.6 equiv; freshly prepared from trimethylsilylmethyl chloride and magnesium) in dry THF $(30 \mathrm{~mL})$ was added dropwise over a period of $1-2 \mathrm{~h}$. The cold mixture was stirred for $1 \mathrm{~h}$ and ester $8(7-10 \mathrm{mmol})$ in dry THF $(10 \mathrm{~mL})$ was added dropwise over $30 \mathrm{~min}$. The resulting mixture was allowed to warm to room temperature and stirred for 3 days. The reaction mixture was then cooled to $-10^{\circ} \mathrm{C}$ and hydrolyzed by the dropwise addition of $1 \mathrm{M}$ hydrochloric acid. The layers were separated and the aqueous layer extracted with diethyl ether. The combined organic layers were dried over $\mathrm{MgSO}_{4}$ and concentrated. The crude product was purified by flash chromatography (cyclohexane/ AcOEt 95:5).

4.3.1. (3'R,4R)-4-( $N$ - $\alpha$-Methylbenzyl- $N^{\prime}$-benzylamino)1-trimethylsilyl-2-trimethylsilylmethylpentan-2-ol 9a. Colourless oil; yield: 90\%; TLC: $R_{\mathrm{f}}=0.52$ (cyclohexane/AcOEt 95:5); de $=82 \%[\alpha]_{\mathrm{D}}^{25}=+24\left(c 1.12, \mathrm{CHCl}_{3}\right)$; IR $\left(\mathrm{CCl}_{4}\right): v\left(\mathrm{~cm}^{-1}\right) 3240 ;{ }^{1} \mathrm{H}$ NMR $\delta$ major diastereoisomer $-0.21(\mathrm{~s}, 9 \mathrm{H}), 0.00(\mathrm{~s}, 9 \mathrm{H}), 0.05(\mathrm{AB}$ system, $2 \mathrm{H}, \Delta v=235 \mathrm{~Hz}, \delta_{\mathrm{A}}=0.35\left(\mathrm{~d}, 1 \mathrm{H}, J_{\mathrm{AB}}=14.6 \mathrm{~Hz}\right)$, $\left.\delta_{\mathrm{B}}=-0.24\left(\mathrm{~d}, 1 \mathrm{H}, J_{\mathrm{AB}}=14.6 \mathrm{~Hz}\right)\right), 0.77(\mathrm{AB}$ system, $2 \mathrm{H}, \Delta v=44 \mathrm{~Hz}, \delta_{\mathrm{A}}=0.82\left(\mathrm{~d}, 1 \mathrm{H}, J_{\mathrm{AB}}=14.8 \mathrm{~Hz}\right)$, $\left.\delta_{\mathrm{B}}=0.72\left(\mathrm{~d}, 1 \mathrm{H}, J_{\mathrm{AB}}=14.8 \mathrm{~Hz}\right)\right), 1.07(\mathrm{~d}, 3 \mathrm{H}$, $J=6.4 \mathrm{~Hz}), 1.35(\mathrm{~d}, 3 \mathrm{H}, J=7.2 \mathrm{~Hz}), 1.48(\mathrm{AB}$ part of $\mathrm{ABX}$ system, 2H, H-3, $\Delta v=385 \mathrm{~Hz}, \delta_{\mathrm{A}}=1.96 \quad(\mathrm{t}$, $\left.J=13.2 \mathrm{~Hz}, \quad J_{\mathrm{AX}}=1.6 \mathrm{~Hz}\right), \quad \delta_{\mathrm{B}}=1.00 \quad(\mathrm{dd}$, $\left.\left.J_{\mathrm{AB}}=14.4 \mathrm{~Hz}, \quad J_{\mathrm{BX}}=2.0 \mathrm{~Hz}\right)\right), 3.23-3.32$ (X part of $\mathrm{ABX}$ system, m, 1H), 3.73 (AB system, 2H, $\Delta v=187 \mathrm{~Hz}, \quad \delta_{\mathrm{A}}=3.97 \quad\left(\mathrm{~d}, \quad 1 \mathrm{H}, \quad J_{\mathrm{AB}}=12.8 \mathrm{~Hz}\right)$, $\left.\delta_{\mathrm{B}}=3.50\left(\mathrm{~d}, 1 \mathrm{H}, J_{\mathrm{AB}}=12.8 \mathrm{~Hz}\right)\right), 3.90(\mathrm{q}, 1 \mathrm{H}$, $J=6.8 \mathrm{~Hz}), 6.29(\mathrm{~s}, 1 \mathrm{H}), 7.10-7.42(\mathrm{~m}, 10 \mathrm{H}) ;{ }^{13} \mathrm{C}$ NMR $\delta$ major diastereoisomer $0.4,1.1,13.6,18.6$ $\left(\mathrm{CH}_{3}\right), 33.1,34.9,45.8\left(\mathrm{CH}_{2}\right), 48.9(\mathrm{CH}), 49.0\left(\mathrm{CH}_{2}\right)$, $56.2(\mathrm{CH}), 75.8(\mathrm{C}), 127.4,128.4,128.7,129.1,129.8$ $(\mathrm{CH}), 139.2,142.6(\mathrm{C})$. Anal. Calcd for $\mathrm{C}_{27} \mathrm{H}_{45} \mathrm{NOSi}_{2}$ : 
C, 71.14; H, 9.95; N, 3.07. Found: C, 71.57; H, 10.08; N, 3.10 .

4.3.2. $\quad\left(3^{\prime} S, 4 S\right)-4-\left(N\right.$ - $\alpha$-Methylbenzyl- $N^{\prime}$-benzylamino)1-trimethylsilyl-2-trimethylsilylmethylheptan-2-ol 9b Yellow oil; yield: $72 \%$; TLC: $R_{\mathrm{f}}=0.38$ (cyclohexane/ AcOEt, 95:5); $[\alpha]_{\mathrm{D}}^{25}=-5\left(c 1.04, \mathrm{CHCl}_{3}\right) ; \mathrm{IR}\left(\mathrm{CCl}_{4}\right): v$ $\left(\mathrm{cm}^{-1}\right)$ 3260; ${ }^{1} \mathrm{H}$ NMR $\delta$ major diastereoisomer -0.15 $(\mathrm{s}, 9 \mathrm{H}), 0.00(\mathrm{~s}, 9 \mathrm{H}), 0.19(\mathrm{AB}$ system, $2 \mathrm{H}, \Delta v=158 \mathrm{~Hz}$, $\delta_{\mathrm{A}}=0.38\left(\mathrm{~d}, 1 \mathrm{H}, J_{\mathrm{AB}}=14.6 \mathrm{~Hz}\right), \delta_{\mathrm{B}}=-0.01(\mathrm{~d}, 1 \mathrm{H}$, $\left.\left.J_{\mathrm{AB}}=14.6 \mathrm{~Hz}\right)\right), 0.86(\mathrm{AB}$ system, $2 \mathrm{H}, \Delta v=32 \mathrm{~Hz}$, $\delta_{\mathrm{A}}=0.90\left(\mathrm{~d}, 1 \mathrm{H}, J_{\mathrm{AB}}=14.6 \mathrm{~Hz}\right), \delta_{\mathrm{B}}=0.82(\mathrm{~d}, 1 \mathrm{H}$, $\left.\left.J_{\mathrm{AB}}=14.6 \mathrm{~Hz}\right)\right), 0.92(\mathrm{t}, 3 \mathrm{H}, J=7.0 \mathrm{~Hz}), 1.13-1.32(\mathrm{~m}$, $2 \mathrm{H}), 1.35(\mathrm{~d}, 3 \mathrm{H}, J=6.8 \mathrm{~Hz}), 1.51(\mathrm{AB}$ part of $\mathrm{ABX}$ system, $2 \mathrm{H}, \quad \Delta v=228 \mathrm{~Hz}, \quad \delta_{\mathrm{A}}=1.80 \quad(\mathrm{dd}, \quad 1 \mathrm{H}$, $\left.J_{\mathrm{AB}}=14.5 \mathrm{~Hz}, \quad J_{\mathrm{AX}}=11.4 \mathrm{~Hz}\right), \quad \delta_{\mathrm{B}}=1.23 \quad(\mathrm{dd}, 1 \mathrm{H}$, $\left.\left.J_{\mathrm{AB}}=14.5 \mathrm{~Hz}, J_{\mathrm{BX}}=2.4 \mathrm{~Hz}\right)\right), 1.65-1.73(\mathrm{~m}, 2 \mathrm{H}, \mathrm{H}-5)$, 3.09 (X part of ABX system, t, $1 \mathrm{H}, J=10.4 \mathrm{~Hz}$ ), 3.75 $\left(\mathrm{AB}\right.$ system, $2 \mathrm{H}, \Delta v=158 \mathrm{~Hz}, \delta_{\mathrm{A}}=3.95(\mathrm{~d}, 1 \mathrm{H}$, $\left.\left.J_{\mathrm{AB}}=13.1 \mathrm{~Hz}\right), \delta_{\mathrm{B}}=3.55\left(\mathrm{~d}, 1 \mathrm{H}, J_{\mathrm{AB}}=13.1 \mathrm{~Hz}\right)\right), 3.91$ $(\mathrm{q}, 1 \mathrm{H}, J=6.8 \mathrm{~Hz}), 6.36(\mathrm{br} \mathrm{s}, 1 \mathrm{H}), 7.11-7.40(\mathrm{~m}$, $10 \mathrm{H}, \mathrm{H}$ aromatics); ${ }^{13} \mathrm{C}$ NMR $\delta$ major diastereoisomer $0.9,1.0,14.7\left(\mathrm{CH}_{3}\right), 21.1,32.0,34.9,35.0,43.4,49.5$ $\left(\mathrm{CH}_{2}\right), 54.3,57.2(\mathrm{CH}), 75.9(\mathrm{C}), 127.3,128.3,128.5$, 128.6, 128.9, $129.6(\mathrm{CH}), 139.5,142.7(\mathrm{C})$; MS (CI, methane) for $\mathrm{C}_{29} \mathrm{H}_{49} \mathrm{NOSi}_{2}+\mathrm{H}: m / z \quad 484\left(\mathrm{M}+\mathrm{H}^{+}\right)$. Anal. Calcd for $\mathrm{C}_{29} \mathrm{H}_{49} \mathrm{NOSi}_{2}$ : C, 71.98; H, 10.21; N, 2.89. Found: C, $72.25 ; \mathrm{H}, 10.30 ; \mathrm{N}, 3.38$.

4.3.3. $\quad\left(3^{\prime} S, 4 S\right)-4-\left(N-\alpha\right.$-Methylbenzyl- $N^{\prime}$-benzylamino $)$ 1-trimethylsilyl-2-trimethylsilylmethyltridecan-2-ol 9c. Yellow oil; yield: 71\%; TLC: $R_{\mathrm{f}}=0.48$ (cyclohexane/ AcOEt 95:5); $[\alpha]_{\mathrm{D}^{25}}=-3\left(c 0.95, \mathrm{CHCl}_{3}\right)$; FTIR (film): $v\left(\mathrm{~cm}^{-1}\right) 3256 ;{ }^{1} \mathrm{H}$ NMR $\delta$ major diastereoisomer $-0.15(\mathrm{~s}, 9 \mathrm{H}), 0.00(\mathrm{~s}, 10 \mathrm{H}), 0.37(\mathrm{~d}, 1 \mathrm{H}), 0.80-0.92$ $(\mathrm{m}, 5 \mathrm{H}), 1.10-1.42(\mathrm{~m}, 17 \mathrm{H}), 1.35(\mathrm{~d}, 3 \mathrm{H}, J=6.9 \mathrm{~Hz})$, $1.80(\mathrm{dd}, 1 \mathrm{H}, J=14.3 \mathrm{~Hz}, J=11.4 \mathrm{~Hz}), 3.1(\mathrm{t}, 1 \mathrm{H}$, $J=9.9 \mathrm{~Hz}), \quad 3.75 \quad(\mathrm{AB}$ system, $2 \mathrm{H}, \quad \Delta v=159 \mathrm{~Hz}$, $\delta_{\mathrm{A}}=3.95\left(\mathrm{~d}, 1 \mathrm{H}, J_{\mathrm{AB}}=13.1 \mathrm{~Hz}\right), \delta_{\mathrm{B}}=3.55(\mathrm{~d}, 1 \mathrm{H}$, $\left.\left.J_{\mathrm{AB}}=13.1 \mathrm{~Hz}\right)\right), 3.90(\mathrm{q}, 1 \mathrm{H}, J=6.9 \mathrm{~Hz}), 6.35(\mathrm{br} \mathrm{s}$, $1 \mathrm{H}), 7.13-7.40(\mathrm{~m}, 10 \mathrm{H}) ;{ }^{13} \mathrm{C}$ NMR $\delta$ major diastereoisomer $0.8,1.0,14.1,14.7\left(\mathrm{CH}_{3}\right), 22.7,26.9,27.9,29.3$, 29.6, 32.0, 35.0, 43.4, $49.4\left(\mathrm{CH}_{2}\right), 54.6,57.2(\mathrm{CH}), 75.9$ (C), 127.1, 127.3, 128.3, 128.5, 128.9, $129.6(\mathrm{CH})$, $139.5,142.7$ (C); MS (CI, methane) for $\mathrm{C}_{35} \mathrm{H}_{61} \mathrm{NO}$ $\mathrm{Si}_{2}+\mathrm{H}: m / z 568\left(\mathrm{M}+\mathrm{H}^{+}\right)$. Anal. Calcd for $\mathrm{C}_{35} \mathrm{H}_{61} \mathrm{NO}-$ $\mathrm{Si}_{2}$ : C, 74.01; H, 10.82; N, 2.47. Found: C, 74.53; H, $11.11 ; \mathrm{N}, 2.65$.

\subsection{General procedure for preparation of $\mathbf{1 0}$}

To a solution of $9(1 \mathrm{~g})$ in methanol $(13 \mathrm{~mL})$, acetic acid $(0.31 \mathrm{~mL})$, water $(2.7 \mathrm{~mL})$ and THF $(2.4 \mathrm{~mL})$ was added Pearlman's catalyst $(0.3 \mathrm{~g})$. The resulting mixture was stirred under $3.5 \mathrm{~atm}$ of hydrogen at room temperature for $24 \mathrm{~h}$ in Parr apparatus. The mixture was filtered through Celite and concentrated to give a residue which was treated with sodium hydrogenocarbonate then extracted with methylene chloride and organic layers were dried $\left(\mathrm{K}_{2} \mathrm{CO}_{3}\right)$ and evaporated.

4.4.1. (4R)-4-Amino-1-trimethylsilyl-2-trimethylsilylmethylpentan-2-ol 10a. Liquid purified by distillation on Kugelrohr: $172{ }^{\circ} \mathrm{C} / 0.5 \mathrm{mmHg}$; yield: $92 \%$; TLC: $R_{\mathrm{f}}=0.18 \quad(\mathrm{AcOEt}) ; \quad[\alpha]_{\mathrm{D}}^{25}=+1 \quad\left(c \quad 1.18, \quad \mathrm{CHCl}_{3}\right)$; IR $\left(\mathrm{CCl}_{4}\right): v\left(\mathrm{~cm}^{-1}\right) 3260 ;{ }^{1} \mathrm{H}$ NMR $0.03(\mathrm{~s}, 1 \mathrm{H}), 0.06$ $(\mathrm{s}, 1 \mathrm{H}), 1.01\left(\mathrm{AB}\right.$ system, $2 \mathrm{H}, \Delta v=42 \mathrm{~Hz}, \delta_{\mathrm{A}}=1.06$ $\left(\mathrm{d}, \quad 1 \mathrm{H}, \quad J_{\mathrm{AB}}=15.0 \mathrm{~Hz}\right), \quad \delta_{\mathrm{B}}=0.96 \quad(\mathrm{~d}, \quad 1 \mathrm{H}$, $\left.\left.J_{\mathrm{AB}}=15.0 \mathrm{~Hz}\right)\right), 1.10(\mathrm{~d}, 3 \mathrm{H}, J=6.4 \mathrm{~Hz}), 1.17(\mathrm{AB}$ system, $2 \mathrm{H}, \Delta v=73 \mathrm{~Hz}, \delta_{\mathrm{A}}=1.26\left(\mathrm{~d}, 1 \mathrm{H}, J_{\mathrm{AB}}=14.3 \mathrm{~Hz}\right)$, $\left.\delta_{\mathrm{B}}=1.08\left(\mathrm{~d}, 1 \mathrm{H}, J_{\mathrm{AB}}=14.3 \mathrm{~Hz}\right)\right), 1.47(\mathrm{AB}$ part of $\mathrm{ABX}$ system, $2 \mathrm{H}, \quad \Delta v=24 \mathrm{~Hz}, \quad \delta_{\mathrm{A}}=1.50 \quad(\mathrm{dd}$, $\left.J_{\mathrm{AB}}=14.3 \mathrm{~Hz}, \quad J_{\mathrm{AX}}=2.6 \mathrm{~Hz}\right), \quad \delta_{\mathrm{B}}=1.44 \quad(\mathrm{dd}$, $\left.\left.J_{\mathrm{AB}}=14.3 \mathrm{~Hz}, J_{\mathrm{BX}}=10.9 \mathrm{~Hz}\right)\right), 3.15(\mathrm{X}$ part of $\mathrm{ABX}$ system, $\mathrm{m}, 1 \mathrm{H}) ;{ }^{13} \mathrm{C}$ NMR $\delta(\mathrm{ppm}) \quad 0.6,1.0,28.1$ $\left(\mathrm{CH}_{3}\right), 32.8,35.0\left(\mathrm{CH}_{2}\right), 45.2(\mathrm{CH}), 50.9\left(\mathrm{CH}_{2}\right), 75.7$ (C); HRMS (ESI) $\mathrm{m} / z$ caled for $\mathrm{C}_{12} \mathrm{H}_{31} \mathrm{NOSi}_{2}+\mathrm{H}$ : 262.2022. Found: $262.2031\left(\mathrm{M}+\mathrm{H}^{+}\right)$.

4.4.2. (4S)-4-Amino-1-trimethylsilyl-2-trimethylsilylmethylheptan-2-ol 10b. Liquid purified by distillation on Kugelrohr: $187^{\circ} \mathrm{C} / 0.5 \mathrm{mmHg}$; yield: $80 \%$; TLC: $\left.R_{\mathrm{f}}=0.33(\mathrm{AcOEt})\right) ;[\alpha]_{\mathrm{D}}^{25}=-3 \quad\left(c 1.11, \mathrm{CHCl}_{3}\right) ; \mathrm{IR}$ $\left(\mathrm{CCl}_{4}\right): v\left(\mathrm{~cm}^{-1}\right) \quad 3300 ;{ }^{1} \mathrm{H} \quad \mathrm{NMR} \quad \delta 0.04(\mathrm{~s}, 9 \mathrm{H})$, $0.06(\mathrm{~s}, 9 \mathrm{H}), 0.92(\mathrm{t}, 3 \mathrm{H}, J=6.8 \mathrm{~Hz}), 1.02$ (AB system, $2 \mathrm{H}, \Delta v=41 \mathrm{~Hz}, \delta_{\mathrm{A}}=1.07\left(\mathrm{~d}, 1 \mathrm{H}, J_{\mathrm{AB}}=14.7 \mathrm{~Hz}\right)$, $\left.\delta_{\mathrm{B}}=0.97\left(\mathrm{~d}, 1 \mathrm{H}, J_{\mathrm{AB}}=14.7 \mathrm{~Hz}\right)\right), 1.15(\mathrm{AB}$ system, $2 \mathrm{H}, \Delta v=83 \mathrm{~Hz}, \delta_{\mathrm{A}}=1.26\left(\mathrm{~d}, 1 \mathrm{H}, \quad J_{\mathrm{AB}}=14.7 \mathrm{~Hz}\right)$, $\left.\delta_{\mathrm{B}}=1.05\left(\mathrm{~d}, 1 \mathrm{H}, J_{\mathrm{AB}}=14.7 \mathrm{~Hz}\right)\right), 1.18-1.46(\mathrm{~m}, 5 \mathrm{H})$, $1.50(\mathrm{dd}, 1 \mathrm{H}, J=14.1 \mathrm{~Hz}, J=2.8 \mathrm{~Hz}), 2.96(\mathrm{~m}, 1 \mathrm{H})$; ${ }^{13} \mathrm{C}$ NMR $\delta 1.0,1.3,14.2\left(\mathrm{CH}_{3}\right), 18.7,32.4,35.1,43.9$, $49.2\left(\mathrm{CH}_{2}\right), 49.4(\mathrm{CH}), 75.6(\mathrm{C})$; MS (CI, methane) $\mathrm{m} /$ $z$ calcd for $\mathrm{C}_{14} \mathrm{H}_{35} \mathrm{NOSi}_{2}+\mathrm{H}: 290\left(\mathrm{M}+\mathrm{H}^{+}\right)$. Anal. Calcd for $\mathrm{C}_{14} \mathrm{H}_{35} \mathrm{NOSi}_{2}$ : C, 58.06; H, 12.18; N, 4.84. Found: C, 57.99; H, 13.52; N, 5.04.

4.4.3. (4S)-4-Amino-1-trimethylsilyl-2-trimethylsily lmethyltridecan-2-ol 10c. Oil; yield: 89\%; TLC: $R_{\mathrm{f}}=0.25$ $(\mathrm{AcOEt}) ; \quad$ ee $=80 \% ;[\alpha]_{\mathrm{D}}^{25}=-2.5 \quad\left(c \quad 1.07, \quad \mathrm{CHCl}_{3}\right)$; IR $\left(\mathrm{CCl}_{4}\right): v\left(\mathrm{~cm}^{-1}\right) 3300 ;{ }^{1} \mathrm{H}$ NMR $\left(400 \mathrm{MHz}, \mathrm{CDCl}_{3}\right)$ : $\delta(\mathrm{ppm}) 0.00(\mathrm{~s}, 9 \mathrm{H}), 0.02(\mathrm{~s}, 9 \mathrm{H}), 0.83(\mathrm{t}, 3 \mathrm{H}$, $J=6.8 \mathrm{~Hz}), \quad 0.98 \quad(\mathrm{AB}$ system, $2 \mathrm{H}, \quad \Delta v=34 \mathrm{~Hz}$, $\delta_{\mathrm{A}}=1.03\left(\mathrm{~d}, 1 \mathrm{H}, J_{\mathrm{AB}}=14.4 \mathrm{~Hz}\right), \delta_{\mathrm{B}}=0.94(\mathrm{~d}, 1 \mathrm{H}$, $\left.\left.J_{\mathrm{AB}}=14.4 \mathrm{~Hz}\right)\right), 0.99(\mathrm{~d}, 1 \mathrm{H}, J=14.3 \mathrm{~Hz}), 1.18-1.27$ $(\mathrm{m}, 15 \mathrm{H}), 1.29-1.36(\mathrm{~m}, 2 \mathrm{H}), 1.44$ (AB part of ABX system, $2 \mathrm{H}, v=21 \mathrm{~Hz}, \delta_{\mathrm{A}}=1.47\left(\mathrm{dd}, 1 \mathrm{H}, J_{\mathrm{AB}}=14.2 \mathrm{~Hz}\right.$, $\left.J_{\mathrm{AX}}=2.2 \mathrm{~Hz}\right), \quad \delta_{\mathrm{B}}=1.41 \quad\left(\mathrm{dd}, \quad 1 \mathrm{H}, \quad J_{\mathrm{AB}}=14.2 \mathrm{~Hz}\right.$, $J_{\mathrm{BX}}=11.5 \mathrm{~Hz}$ )), 2.91 (partie $\mathrm{X}$ de systeme $\mathrm{ABX}, \mathrm{m}$, 1H), 3.21 (br s, $2 \mathrm{H}) ;{ }^{13} \mathrm{C}$ NMR $\left(100 \mathrm{MHz}, \mathrm{CDCl}_{3}\right): \delta$ (ppm) 0.8, 1.0, $14.8\left(\mathrm{CH}_{3}\right), 22.8,25.7,29.4,29.6,29.7$, $32.0,32.4,35.2,41.0,49.3\left(\mathrm{CH}_{2}\right), 49.5(\mathrm{CH}), 75.8(\mathrm{C})$; HRMS (EI) $m / z$ calcd for $\mathrm{C}_{20} \mathrm{H}_{47} \mathrm{NOSi}_{2}-\mathrm{CH}_{2} \mathrm{SiMe}_{3}$ : 286.2566. Found: $286.2554\left(\mathrm{M}-\mathrm{CH}_{2} \mathrm{SiMe}_{3}{ }^{+}\right)$.

\section{5. (2R)-4-Trimethylsilylmethylpent-4-en-2-amine 5a}

A solution of $\mathrm{HCl} 1 \mathrm{M}$ (4.4 equiv) was added slowly to a cooled $\left(0^{\circ} \mathrm{C}\right) 0.5 \mathrm{M}$ solution of $(R)-10 \mathrm{a}$ in diethylether and stirred at $0^{\circ} \mathrm{C}$ for $1 \mathrm{~h}$. The excess of acid was neutralized with a saturated solution of sodium hydrogenocarbonate and the aqueous phase was extracted with ether. The organic phase was dried $\left(\mathrm{K}_{2} \mathrm{CO}_{3}\right)$ and concentrated under atmospheric pressure. Liquid; yield: $93 \% ;$ TLC: $R_{\mathrm{f}}=0.07(\mathrm{AcOEt}) ;$ ee $=82 \% ;[\alpha]_{\mathrm{D}}^{25}=+20$ (c $\left.0.88, \mathrm{CHCl}_{3}\right) ; \mathrm{Bp}=43{ }^{\circ} \mathrm{C}(1 \mathrm{mmHg}) ; \mathrm{IR}\left(\mathrm{CCl}_{4}\right): v$ $\left(\mathrm{cm}^{-1}\right)$ 3080, 1630; ${ }^{1} \mathrm{H}$ NMR $\delta 0.00(\mathrm{~s}, 9 \mathrm{H}), 1.07(\mathrm{~d}$, 
$3 \mathrm{H}, J=6.4 \mathrm{~Hz}$ ), 1.35 (br s, 2H), 1.51 (AB system, 2H, $\Delta v=13 \mathrm{~Hz}, \quad \delta_{\mathrm{A}}=1.52 \quad\left(\mathrm{~d}, \quad 1 \mathrm{H}, \quad J_{\mathrm{AB}}=13.4 \mathrm{~Hz}\right)$, $\left.\delta_{\mathrm{B}}=1.49\left(\mathrm{~d}, 1 \mathrm{H}, J_{\mathrm{AB}}=13.4 \mathrm{~Hz}\right)\right), 1.94(\mathrm{AB}$ part of $\mathrm{ABX}$ system $\mathrm{ABX}, 2 \mathrm{H}, \Delta v=70 \mathrm{~Hz}, \delta_{\mathrm{A}}=2.03(\mathrm{dd}, 1 \mathrm{H}$, $\left.J_{\mathrm{AB}}=13.6 \mathrm{~Hz}, \quad J_{\mathrm{AX}}=4.6 \mathrm{~Hz}\right), \quad \delta_{\mathrm{B}}=1.86(\mathrm{dd}, \quad 1 \mathrm{H}$, $\left.\left.J_{\mathrm{AB}}=13.6 \mathrm{~Hz}, J_{\mathrm{BX}}=8.8 \mathrm{~Hz}\right)\right), 3.05(\mathrm{~m}, 1 \mathrm{H}), 4.60(\mathrm{~s}$, $1 \mathrm{H}), 4.62(\mathrm{~s}, 1 \mathrm{H}) ;{ }^{13} \mathrm{C}$ NMR $\delta-1.3,23.9\left(\mathrm{CH}_{3}\right), 26.5$ $\left(\mathrm{CH}_{2}\right), 44.6(\mathrm{CH}), 49.4,109.6\left(\mathrm{CH}_{2}\right), 145.3(\mathrm{C})$; HRMS (ESI) $\mathrm{m} / z$ calcd for $\mathrm{C}_{9} \mathrm{H}_{21} \mathrm{NSi}+\mathrm{H}: 172.1521$. Found: $172.1518\left(\mathrm{M}+\mathrm{H}^{+}\right)$.

\subsection{General procedure for preparation of 4-methyl- enepiperidines}

4.6.1. From aminoallylsilane $(\boldsymbol{R})-5 \mathbf{a}$. To a solution of the aminoallylsilane $(R)-\mathbf{5 a}$ in a mixture of THF and water $\left(1: 1 \mathrm{v} / \mathrm{v} ; 0.73 \mathrm{~mol} \mathrm{~L}^{-1}\right)$ was added at ambient temperature aldehyde or cyclohexanone (1.2 equiv). The solution was stirred for $20 \mathrm{~min}$ and trifluoroacetic acid (1.1 equiv) was added dropwise. After stirring for one day, the reaction mixture was neutralized with a saturated solution of sodium hydrogenocarbonate and the aqueous phase was extracted with ether. The organic layers were collected, dried $\left(\mathrm{K}_{2} \mathrm{CO}_{3}\right)$ and evaporated at atmospheric pressure. The crude product was purified by flash chromatography (elution gradient: pentane then pentane/diethylether)

4.6.2. From $\beta$-aminohydroxysilanes $(R)$-10a, $(S)$-10b and $(S)-10 c$. To a solution of aminoalcohol $(S)$-10b and $(S)-10 \mathrm{c}$ in a mixture of THF and water (1:1; $0.76 \mathrm{~mol} \mathrm{~L}^{-1}$ ) was added at room temperature the carbonyl compound. The solution was stirred for $24 \mathrm{~h}$ and trifluoroacetic acid (10 equiv) added. One day after the addition, the acid was neutralized with a saturated solution of sodium hydrogenocarbonate. The aqueous phase was extracted with diethyl ether. The organic layers were collected, dried over $\mathrm{K}_{2} \mathrm{CO}_{3}$ and evaporated at atmospheric pressure. The crude product was purified by flash chromatography (elution gradient: pentane then pentane/diethyl ether).

4.6.3. (2R,6S)-2-Methyl-4-methylen-6-propylpiperidine 17a. Liquid. (a) From aminoallylsilane $(R)-5 a$. Yield: $49 \%$; TLC: $R_{\mathrm{f}}=0.38 \quad\left(\mathrm{AcOEt}+\right.$ drops of $\mathrm{NH}_{4} \mathrm{OH}$ $28 \%) ; \quad[\alpha]_{\mathrm{D}}^{21}=-4 \quad\left(c 1.05, \mathrm{CHCl}_{3}\right) ; \operatorname{FTIR}$ (film): v $\left(\mathrm{cm}^{-1}\right) \quad 3071, \quad 1651 ;{ }^{1} \mathrm{H} \quad \mathrm{NMR} \quad \delta \quad 0.87 \quad(\mathrm{t}, \quad 3 \mathrm{H}$, $J=7.0 \mathrm{~Hz}), 1.06(\mathrm{~d}, 3 \mathrm{H}, J=6.2 \mathrm{~Hz}), 1.26-1.39(\mathrm{~m}$, $4 \mathrm{H}), 1.53(\mathrm{br} \mathrm{s}, 1 \mathrm{H}), 1.67(\mathrm{t}, 1 \mathrm{H}, J=13.4 \mathrm{~Hz}), 1.70(\mathrm{t}$, $1 \mathrm{H}, J=13.4 \mathrm{~Hz}), 2.15(\mathrm{~d}, 1 \mathrm{H}, J=13.4 \mathrm{~Hz}), 2.20(\mathrm{~d}$, $1 \mathrm{H}, J=13.4 \mathrm{~Hz}), 2.42-2.50(\mathrm{~m}, 1 \mathrm{H}), 2.52-2.62(\mathrm{~m}$, $1 \mathrm{H}), 4.59(\mathrm{~s}, 2 \mathrm{H}) ;{ }^{13} \mathrm{C}$ NMR $\delta 14.2\left(\mathrm{CH}_{3}\right), 19.1\left(\mathrm{CH}_{2}\right)$, $22.7\left(\mathrm{CH}_{3}\right), 39.3,41.3,43.4\left(\mathrm{CH}_{2}\right), 52.3,57.5(\mathrm{CH})$, $107.6\left(\mathrm{CH}_{2}\right), 146.9$ (C); HRMS (ESI) $\mathrm{m} / \mathrm{z}$ calcd for $\mathrm{C}_{10} \mathrm{H}_{19} \mathrm{~N}+\mathrm{H}$ : 154.1596 . Found: $154.1591\left(\mathrm{M}+\mathrm{H}^{+}\right)$. (b) From aminohydroxysilane (R)-10a. Yield: $53 \%$; $[\alpha]_{\mathrm{D}}^{21}=-5.5\left(c 0.94, \mathrm{CHCl}_{3}\right)_{21}$ (c) From aminohydroxysilane $(S)$-10b. Yield: $34 \% ;[\alpha]_{\mathrm{D}}^{21}=-5.5\left(c 0.93, \mathrm{CHCl}_{3}\right)$.

4.6.4. (2R,6R)-2-Methyl-4-methylen-6-propenylpiperidine 18a. From aminoallylsilane $(R)-5$ a. Liquid, yield: $39 \%$; TLC: $R_{\mathrm{f}}=0.56$ (AcOEt + drops of $\mathrm{NH}_{4} \mathrm{OH} 28 \%$ ); $[\alpha]_{\mathrm{D}}^{21}=-4\left(c 0.86, \mathrm{CHCl}_{3}\right)$; ee $=78 \%$; FTIR (film): $v$ $\left(\mathrm{cm}^{-1}\right) \quad 3071, \quad 1651 ;{ }^{1} \mathrm{H} \quad \mathrm{NMR} \quad \delta \quad 1.08 \quad(\mathrm{~d}, \quad 3 \mathrm{H}$, $J=6.2 \mathrm{~Hz}), 1.63(\mathrm{~d}, 3 \mathrm{H}, J=6.3 \mathrm{~Hz}), 1.72(\mathrm{t}, 1 \mathrm{H}$, $J=12.5 \mathrm{~Hz}), 1.86(\mathrm{t}, 1 \mathrm{H}, J=12.2 \mathrm{~Hz}), 2.13-2.17(2 \mathrm{H}$, $\mathrm{m}), 2.58-2.66(\mathrm{~m}, 1 \mathrm{H}), 3.01(\mathrm{ddd}, 1 \mathrm{H}, J=11.3 \mathrm{~Hz}$, $J=6.9 \mathrm{~Hz}, J=2.6 \mathrm{~Hz}), 4.63(2 \mathrm{H}, \mathrm{s}), 5.43(\mathrm{ddd}, 1 \mathrm{H}$, $J=15.3 \mathrm{~Hz}, J=6.9 \mathrm{~Hz}, J=1.4 \mathrm{~Hz}), 5.58(\mathrm{dq}, 1 \mathrm{H}, J=$ $6.3 \mathrm{~Hz}, J=15.3 \mathrm{~Hz}) ;{ }^{13} \mathrm{C}$ NMR $\delta 17.8,22.6\left(\mathrm{CH}_{3}\right)$, 41.3, $42.9\left(\mathrm{CH}_{2}\right), 53.0,60.1(\mathrm{CH}), 108.0\left(\mathrm{CH}_{2}\right), 125.8$, $134.1(\mathrm{CH}), 146.3$ (C); HRMS (ESI) $\mathrm{m} / \mathrm{z}$ calcd for $\mathrm{C}_{10} \mathrm{H}_{17} \mathrm{~N}+\mathrm{H}$ : 152.1439 . Found: $152.1457\left(\mathrm{M}+\mathrm{H}^{+}\right)$.

4.6.5. (2R,6S)-2-Methyl-4-methylen-6-nonylpiperidine 19a. Liquid. (a) From aminoallylsilane $(R)-5 a$. Yield: $49 \%$; TLC: $R_{\mathrm{f}}=0.44 \quad$ (AcOEt + drops of $\mathrm{NH}_{4} \mathrm{OH}$ $28 \%) ;[\alpha]_{\mathrm{D}}^{21}=-1.5\left(c 0.94, \mathrm{CHCl}_{3}\right) ;$ ee $=28 \% \quad$ FTIR (film): $v\left(\mathrm{~cm}^{-1}\right) 3071,1651 ;{ }^{1} \mathrm{H}$ NMR $\delta 0.83(\mathrm{t}, 3 \mathrm{H}$, $J=7.0 \mathrm{~Hz}), 1.07(\mathrm{~d}, 3 \mathrm{H}, J=6.2 \mathrm{~Hz}), 1.14-1.46(\mathrm{~m}$, $16 \mathrm{H}), 1.67 \quad(\mathrm{t}, \quad 1 \mathrm{H}, \quad J=12.8 \mathrm{~Hz}), 1.70 \quad(\mathrm{t}, \quad 1 \mathrm{H}$, $J=12.8 \mathrm{~Hz}), 2.16(\mathrm{~d}, 1 \mathrm{H}, J=13.2 \mathrm{~Hz}), 2.19(\mathrm{~d}, 1 \mathrm{H}$, $J=13.2 \mathrm{~Hz}), 2.41-2.49(\mathrm{~m}, 1 \mathrm{H}), 2.53-2.62(\mathrm{~m}, 1 \mathrm{H})$, $4.59(\mathrm{~s}, 2 \mathrm{H}) ;{ }^{13} \mathrm{C}$ NMR $\delta 14.1\left(\mathrm{CH}_{3}\right), 22.8\left(\mathrm{CH}_{3}\right), 22.7$, 26.0, 29.3, 29.6, 29.7, 29.8, 31.9, 37.2, 41.3, $43.5\left(\mathrm{CH}_{2}\right)$, 53.3, $57.9(\mathrm{CH}), 107.6\left(\mathrm{CH}_{2}\right), 146.9(\mathrm{C})$; HRMS (EI) $m / z$ calcd for $\mathrm{C}_{16} \mathrm{H}_{31} \mathrm{~N}-\mathrm{CH}_{3}$ : 222.2222. Found: $222.2200\left(\mathrm{M}-\mathrm{CH}_{3}{ }^{+}\right)$. (b) From aminoalcohol $(R)-10 a$. Yield: $73 \% ;[\alpha]_{\mathrm{D}}^{21}=-4.5\left(c 0.98, \mathrm{CHCl}_{3}\right)$; ee $=74 \%$. (c) From aminohydroxysilane (S)-10c. Yield: 53\%; $[\alpha]_{\mathrm{D}}^{21}=-6\left(c 1.00, \mathrm{CHCl}_{3}\right)$; ee $=76 \%(\operatorname{method} \mathrm{A})$.

4.6.6. (2R,6S)-2-Methyl-4-methylen-6-undecylpiperidine 20a. Liquid (a) From aminoallylsilane $(R)-5$ a. Yield: $58 \%$; TLC: $R_{\mathrm{f}}=0.56 \quad$ (AcOEt + drops of $\mathrm{NH}_{4} \mathrm{OH}$ $28 \%) ;[\alpha]_{\mathrm{D}}^{21}=-2.5\left(\right.$ c $\left.1.16, \mathrm{CHCl}_{3}\right) ;$ ee $=38 \%$; FTIR (film): $v\left(\mathrm{~cm}^{-1}\right) 3071,1651 ;{ }^{1} \mathrm{H}$ NMR $\delta 0.86(\mathrm{t}, 3 \mathrm{H}$, $J=6.6 \mathrm{~Hz}), 1.09(\mathrm{~d}, 3 \mathrm{H}, J=6.2 \mathrm{~Hz}), 1.20-1.43(\mathrm{~m}$, $20 \mathrm{H}), 1.70 \quad(\mathrm{t}, \quad 1 \mathrm{H}, \quad J=13.2 \mathrm{~Hz}), 1.74 \quad(\mathrm{t}, \quad 1 \mathrm{H}$, $J=13.2 \mathrm{~Hz}), 2.19(\mathrm{~d}, 1 \mathrm{H}, J=12.7 \mathrm{~Hz}), 2.22(\mathrm{~d}, 1 \mathrm{H}$, $J=12.7 \mathrm{~Hz}), 2.43-2.51(\mathrm{~m}, 1 \mathrm{H}), 2.55-2.65(\mathrm{~m}, 1 \mathrm{H})$, $4.62(\mathrm{~s}, 2 \mathrm{H}) ;{ }^{13} \mathrm{C}$ NMR $\delta 14.1,22.7\left(\mathrm{CH}_{3}\right), 22.8,26.0$, 29.4, 29.6, 29.7, 29.8, 31.9, 37.2, 41.4, $43.5\left(\mathrm{CH}_{2}\right)$, 53.3, $57.9(\mathrm{CH}), 107.6\left(\mathrm{CH}_{2}\right), 147.0(\mathrm{C})$; HRMS (EI) $\mathrm{m} / \mathrm{z}$ calcd for $\mathrm{C}_{18} \mathrm{H}_{35} \mathrm{~N}: 265.2769$. Found: $265.2765\left(\mathrm{M}^{+}\right)$. (b) From aminohydroxysilane $(R)-10 \mathrm{a}$. Yield: $70 \%$; ee $=64 \%$.

4.6.7. (2R,6R)-2-Methyl-4-methylen-6-phenylpiperidine 21a. Liquid (a) From aminoallylsilane $(R)-5$ a. Yield: $70 \%$; TLC: $R_{\mathrm{f}}=0.85 \quad\left(\mathrm{AcOEt}+\right.$ drops of $\mathrm{NH}_{4} \mathrm{OH}$ $28 \%) ;[\alpha]_{\mathrm{D}}^{21}=-9.5\left(\right.$ c $\left.1.12, \mathrm{CHCl}_{3}\right) ;$ ee $=74 \% ;$ FTIR (film): $v\left(\mathrm{~cm}^{-1}\right) 3071,1651 ;{ }^{1} \mathrm{H}$ NMR $\delta 1.12(\mathrm{~d}, 3 \mathrm{H}$, $J=6.4 \mathrm{~Hz}), 1.60($ br s, $1 \mathrm{H}), 1.85(\mathrm{t}, 1 \mathrm{H}, J=12.9 \mathrm{~Hz})$, $2.14(\mathrm{t}, 1 \mathrm{H}, J=12.8 \mathrm{~Hz}), 2.22(1 \mathrm{H}, \mathrm{d}, J=12.8 \mathrm{~Hz})$, $2.32(1 \mathrm{H}, \mathrm{d}, J=12.8 \mathrm{~Hz}), 2.70-2.77(\mathrm{~m}, 1 \mathrm{H}), 3.59(\mathrm{dd}$, $1 \mathrm{H}, J=2.7 \mathrm{~Hz}, J=11.4 \mathrm{~Hz}), 4.67-4.69(2 \mathrm{H}, \mathrm{m}), 7.20$ $7.35(\mathrm{~m}, 5 \mathrm{H}) ;{ }^{13} \mathrm{C}$ NMR $\delta 22.7\left(\mathrm{CH}_{3}\right), 42.9,43.3$ $\left(\mathrm{CH}_{2}\right), 53.7,62.9(\mathrm{CH}), 108.2\left(\mathrm{CH}_{2}\right), 126.7,127.2$, $128.4(\mathrm{CH}), 144.5$. 146.7 (C); HRMS (EI) $\mathrm{m} / \mathrm{z}$ calcd for $\mathrm{C}_{13} \mathrm{H}_{17} \mathrm{~N}$ : 187.1361. Found: $187.1370\left(\mathrm{M}^{+}\right)$. (b) From aminohydroxysilane $(R)-10 \mathrm{a}$. Yield: $70 \% ;[\alpha]_{\mathrm{D}}^{21}=-11.5$ (c $1.09, \mathrm{CHCl}_{3}$ ); ee $=84 \%$.

4.6.8. (2R)-Methyl-4-methylen-1-aza-spiro[5,5]undecane 22. Liquid (a) From aminoallylsilane $(R)-5$ a. Yield: 
$46 \%$; TLC: $R_{\mathrm{f}}=0.55\left(\mathrm{AcOEt}+\right.$ drops of $\mathrm{NH}_{4} \mathrm{OH} 28 \%$ ); $[\alpha]_{\mathrm{D}}^{21}=0 \quad\left(c 1.04, \mathrm{CHCl}_{3}\right)$; ee $=24 \%$; FTIR (film): $v$ $\left(\mathrm{cm}^{-1}\right) 3071,1651 ;{ }^{1} \mathrm{H}$ NMR $\delta 1.03(\mathrm{~d}, 3 \mathrm{H}, J=6.2 \mathrm{~Hz})$, $1.28-1.55(\mathrm{~m}, 11 \mathrm{H}), 1.65(\mathrm{t}, 1 \mathrm{H}, J=10.7 \mathrm{~Hz}), 1.77(\mathrm{~d}$, $1 \mathrm{H}, J=12.7 \mathrm{~Hz}), 2.15-2.23(2 \mathrm{H}, \mathrm{m}), 2.80-2.88(\mathrm{~m}, 1 \mathrm{H})$, $4.564 .58(\mathrm{~m}, 1 \mathrm{H}), 4.66-4.68(\mathrm{~m}, 1 \mathrm{H}) ;{ }^{13} \mathrm{C}$ NMR $\delta 21.8$, 21.9, 31.7, $40.9\left(\mathrm{CH}_{2}\right), 23.1\left(\mathrm{CH}_{3}\right), 43.9,44.8\left(\mathrm{CH}_{2}\right)$, $46.1(\mathrm{CH}), 52.9(\mathrm{C}), 108.8\left(\mathrm{CH}_{2}\right), 145.1(\mathrm{C})$; HRMS (EI) $\mathrm{m} / z$ calcd for $\mathrm{C}_{12} \mathrm{H}_{21} \mathrm{~N}: 179.1674$. Found: 179.1667 $\left(\mathrm{M}^{+}\right)$. (b) From aminohydroxysilane $(R)-10 \mathrm{a}$. Yield: $25 \% ;[\alpha]_{\mathrm{D}}^{21}=0\left(c 0.98, \mathrm{CHCl}_{3}\right) ; \mathrm{ee}=14 \%$.

\subsection{General procedure for preparation of 24}

To a solution of aminohydroxysilane $(R)-10 \mathrm{a}$ in a mixture of THF and water $\left(1: 1 ; 0.76 \mathrm{~mol} \mathrm{~L}^{-1}\right)$ was added at room temperature the carbonyl compound. The mixture was stirred $24 \mathrm{~h}$ and extracted with diethyl ether. The organic layers were collected, dried over $\mathrm{K}_{2} \mathrm{CO}_{3}$ and concentrated. The crude product was purified by flash chromatography (elution gradient: cyclohexane then cyclohexane/AcOEt).

4.7.1. (4R)-4-Methyl-2-nonyl-6,6-bis-trimethylsilylmethyl-[1,3]-oxazinane 24a. Oil; yield: 79\%; TLC: $R_{\mathrm{f}}=0.64$ (AcOEt/cyclohexane 1:9); $[\alpha]_{\mathrm{D}}^{25}=+17.5(c$ $\left.1.08, \mathrm{CHCl}_{3}\right)$; ee $=82 \% ;{ }^{1} \mathrm{H}$ NMR $\delta 0.05(\mathrm{~s}, 9 \mathrm{H}), 0.06$ $(\mathrm{s}, 9 \mathrm{H}), 0.88(\mathrm{t}, 3 \mathrm{H}, J=6.8 \mathrm{~Hz}), 0.93(\mathrm{~d}, 1 \mathrm{H}$, $J=11.2 \mathrm{~Hz}), 1.04(\mathrm{~d}, 3 \mathrm{H}, J=6.4 \mathrm{~Hz}), 1.07-1.51(\mathrm{~m}$, $21 \mathrm{H}) ; 2.88-2.97(\mathrm{~m}, 1 \mathrm{H}), 4.26(\mathrm{t}, 1 \mathrm{H}, J=5.2 \mathrm{~Hz}) ;{ }^{13} \mathrm{C}$ NMR $\delta 0.8,1.2,14.2,22.8\left(-\mathrm{CH}_{3}\right), 22.7,25.1,26.4$, 29.4, 29.6, 29.8, 32.0, 35.0, $36.7\left(\mathrm{CH}_{2}\right), 46.2(\mathrm{CH}), 47.3$ $\left(\mathrm{CH}_{2}\right), 76.7$ (C), $80.8 \quad(\mathrm{CH}) ; \quad \mathrm{MS}$ (ESI) $\mathrm{m} / \mathrm{z}$ for $\mathrm{C}_{22} \mathrm{H}_{49} \mathrm{NOSi}_{2}: 400\left(\mathrm{M}+\mathrm{H}^{+}\right)$.

4.7.2. (4R)-4-Methyl-2-phenyl-6,6-bis-trimethylsilylmethyl-[1,3]-oxazinane 24b. Oil; yield: $59 \%$; TLC: $R_{\mathrm{f}}=0.70($ AcOEt $/$ cyclohexane $2: 8) ;[\alpha]_{\mathrm{D}}^{25}=-5(c 0.91$, $\left.\mathrm{CHCl}_{3}\right)$; ee $=82 \% ;{ }^{1} \mathrm{H}$ NMR $\delta 0.00(\mathrm{~s}, 9 \mathrm{H}), 0.04(\mathrm{~s}$, $9 \mathrm{H}), 1.01 \quad(\mathrm{~d}, \quad 1 \mathrm{H}, \quad J=14.7 \mathrm{~Hz}), 1.05 \quad(\mathrm{~d}, 3 \mathrm{H}$, $J=6.4 \mathrm{~Hz}), \quad 1.15-1.24 \quad(\mathrm{~m}, 3 \mathrm{H}), 1.37 \quad(\mathrm{~d}, 1 \mathrm{H}$, $J=14.8 \mathrm{~Hz}), 1.51(\mathrm{dd}, 1 \mathrm{H}, J=2.9 \mathrm{~Hz}, J=13.2 \mathrm{~Hz})$, $5.27(\mathrm{~s}, 1 \mathrm{H}), 7.19-7.46(\mathrm{~m}, 5 \mathrm{H}) ;{ }^{13} \mathrm{C}$ NMR $\delta 0.9,1.2$, $22.7\left(-\mathrm{CH}_{3}\right), 26.3,35.0\left(\mathrm{CH}_{2}\right), 46.6(\mathrm{CH}), 47.2\left(\mathrm{CH}_{2}\right)$, $77.8(\mathrm{C}), 82.3(\mathrm{CH}), 126.3,128.1,128.8(\mathrm{CH}), 141.5$ (C); MS (ESI) $m / z$ for $\mathrm{C}_{19} \mathrm{H}_{34} \mathrm{NOSi}_{2}: 350\left(\mathrm{M}+\mathrm{H}^{+}\right)$.

\subsection{General procedure for oxidation of 4-methylenepi- peridines $19 \mathrm{a}$ and $19 \mathrm{~b}$}

To a solution of 4-methylenepiperidine $19 \mathbf{a}$ or $19 \mathrm{~b}$ prepared from aminohydroxysilane $(R)-10 \mathrm{a}(2.3-2.5 \mathrm{mmol})$ in aqueous acetic acid solution $\left(80 \%, 0.09 \mathrm{~mol} \mathrm{~L}^{-1}\right)$ was added sodium paraperiodate $\left(\mathrm{Na}_{3} \mathrm{H}_{3} \mathrm{IO}_{6}, 2.2\right.$ equiv) and a crystal of osmium tetroxide. The mixture was stirred for $22 \mathrm{~h}$ at $10^{\circ} \mathrm{C}$ and acetic acid then evaporated under vacuum. A saturated solution of sodium hydrogenocarbonate was added and the aqueous phase extracted with dichloromethane. The organic layers were collected, dried over $\mathrm{MgSO}_{4}$ and evaporated. The crude product was purified by flash chromatography (elution gradient: AcOEt/cyclohexane, 25:75-1:1).
4.8.1. (2R,6S)-2-Methyl-6-nonylpiperidin-4-one 25 . White solid; yield: $68 \%$; TLC: $R_{\mathrm{f}}=0.51(\mathrm{AcOEt}+$ drops of $\left.\mathrm{NH}_{4} \mathrm{OH} 28 \%\right) ;[\alpha]_{\mathrm{D}}^{25}=-1\left(c 0.79, \mathrm{CHCl}_{3}\right)\left\{\right.$ lit. ${ }^{13 \mathrm{~b}}$ $\left.[\alpha]_{\mathrm{D}}^{25}=-1.1 \quad\left(c 1.56, \mathrm{CHCl}_{3}\right)\right\} ; \mathrm{mp}=25-28^{\circ} \mathrm{C}$ (lit. ${ }^{13 \mathrm{~b}}$ $\left.\mathrm{mp}=29-30^{\circ} \mathrm{C}\right) ; \quad$ FTIR $(\mathrm{KBr}): \quad v \quad\left(\mathrm{~cm}^{-1}\right) \quad 1720 ;{ }^{1} \mathrm{H}$ NMR $\delta 0.83(\mathrm{t}, 3 \mathrm{H}, \quad J=6.8 \mathrm{~Hz}), 1.19(\mathrm{~d}, 3 \mathrm{H}$, $J=6.2 \mathrm{~Hz}), 1.23-1.56(\mathrm{~m}, 17 \mathrm{H}), 2.01-2.11(\mathrm{~m}, 2 \mathrm{H})$, 2.32-2.41 (m, 2H), 2.81-2.87 (m, 1H), 2.93-3.01 (m, $1 \mathrm{H}) ;{ }^{13} \mathrm{C}$ NMR $\delta 14.2,22.7\left(\mathrm{CH}_{3}\right), 22.7,25.8,29.4$, 29.6, 29.7, 31.9, 37.1, 48.2, 50.2 $\left(\mathrm{CH}_{2}\right), \quad 52.2$, $56.7(\mathrm{CH}), 210.0(\mathrm{C})$; MS (EI) $\mathrm{m} / z$ for $\mathrm{C}_{15} \mathrm{H}_{29} \mathrm{NO}: 239$ $\left(\mathrm{M}^{+}\right)$.

4.8.2. (2R,6S)-2-Methyl-6-undecylpiperidin-4-one 27. White solid; yield: $67 \%$; TLC: $R_{\mathrm{f}}=0.55(\mathrm{AcOEt}+$ drops of $\left.\mathrm{NH}_{4} \mathrm{OH} \quad 28 \%\right) ; \quad[\alpha]_{\mathrm{D}}^{25}=-1.5 \quad\left(c \quad 0.89, \mathrm{CHCl}_{3}\right)$; $\mathrm{mp}=38-40^{\circ} \mathrm{C} ;$ FTIR $(\mathrm{KBr}): v\left(\mathrm{~cm}^{-1}\right) 1720 ;{ }^{1} \mathrm{H}$ NMR $\delta 0.87(\mathrm{t}, 3 \mathrm{H}, J=6.8 \mathrm{~Hz}), 1.21(\mathrm{~d}, 3 \mathrm{H}, J=6.4 \mathrm{~Hz})$, $1.22-1.56(\mathrm{~m}, 20 \mathrm{H}), 2.01-2.12(\mathrm{~m}, 2 \mathrm{H}), 2.17$ (br s, $1 \mathrm{H})$, 2.30-2.39 (m, 2H), 2.79-2.87 (m, 1H), 2.92-3.01 (m, $1 \mathrm{H}) ;{ }^{13} \mathrm{C}$ NMR $\delta 14.2,22.6\left(\mathrm{CH}_{3}\right), 22.7,25.7,29.5$, 29.5, 29.6, 31.9, 37.0, 48.1, $50.1 \quad\left(\mathrm{CH}_{2}\right), \quad 52.2$, $56.6(\mathrm{CH}), 209.5(\mathrm{C})$; MS (EI) $m / z$ for $\mathrm{C}_{17} \mathrm{H}_{33} \mathrm{NO}: 267$ $\left(\mathrm{M}^{+}\right)$.

\section{9. (2R,4S,6S)-2-Methyl-6-nonylpiperidin-4-ol, (+)- alkaloid 241D 4}

To a solution of piperidin-4-one $25(0.15 \mathrm{~g}, 0.63 \mathrm{mmol})$ in methanol $(3.7 \mathrm{~mL})$ was added sodium borohydride $(0.04 \mathrm{~g}, 1.08 \mathrm{mmol}, 1.7$ equiv). The mixture was stirred for $10 \mathrm{~min}$ and a saturated solution of ammonium chloride added. The aqueous phase was extracted with dichloromethane. The organic layers were collected, dried over $\mathrm{MgSO}_{4}$ and evaporated. The crude product was purified by flash chromatography (elution gradient: AcOEt then AcOEt/methanol 9:1) to give $0.10 \mathrm{~g}$ of 4 . White solid; yield: $66 \%$; TLC: $R_{\mathrm{f}}=0.33$ (AcOEt + drops of $\left.\mathrm{NH}_{4} \mathrm{OH} 28 \%\right) ;[\alpha]_{\mathrm{D}}^{25}=+5.5(c 1.04, \mathrm{MeOH})\left\{\right.$ lit. ${ }^{13 \mathrm{a}}$ $\left.[\alpha]_{\mathrm{D}}^{25}=+6.5 \quad(\mathrm{c} 2, \mathrm{MeOH})\right\} ;$ ee $=74 \% ; \mathrm{mp}=106^{\circ} \mathrm{C}$ (lit. ${ }^{13 \mathrm{a}} \mathrm{mp}=108-109^{\circ} \mathrm{C}$ ); spectral data are identical to literature, ${ }^{13 \mathrm{a}}$ MS (ESI) $\mathrm{m} / \mathrm{z}$ for $\mathrm{C}_{15} \mathrm{H}_{31} \mathrm{NO}+\mathrm{H}: 242$ $\left(\mathrm{M}+\mathrm{H}^{+}\right)$.

\subsection{0. (7R,9S)-7-Methyl-9-undecyl-1,4-dithia-8-aza- spiro[4.5]decane 28}

To a solution of piperidin-4-one $27(0.20 \mathrm{~g}, 0.75 \mathrm{mmol})$ in dry dichloromethane $(1.25 \mathrm{~mL})$ was added ethanedithiol $(0.71 \mathrm{~g}, 7.5 \mathrm{mmol}, 10$ equiv) and boron trifluoride etherate $(0.24 \mathrm{~mL}, 1.89 \mathrm{mmol}, 2.5$ equiv). The mixture was stirred for $20 \mathrm{~h}$ and hydrolyzed with a solution of sodium hydroxide $1 \mathrm{M}$. The aqueous phase was extracted with dichloromethane. The organic layers were collected, washed with a solution of sodium hydroxide $1 \mathrm{M}$, dried over $\mathrm{MgSO}_{4}$ and evaporated. The crude product was purified by flash chromatography (AcOEt) to give $0.21 \mathrm{~g}$ of 28 . Liquid; yield: $82 \%$; TLC: $R_{\mathrm{f}}=0.42$ (AcOEt); $[\alpha]_{\mathrm{D}}^{25}=+6(c)$ $\left\{\right.$ lit. ${ }^{14 \mathrm{c}}[\alpha]_{\mathrm{D}}^{25}=+7.36\left(\mathrm{c} 1.3, \mathrm{CHCl}_{3}\right)$; ; spectral data are identical to literature, ${ }^{14 c}$ MS (EI) $m / z$ for $\mathrm{C}_{19} \mathrm{H}_{37} \mathrm{NS}_{2}$ : $343\left(\mathrm{M}^{+}\right)$. 
4.11. (2R,6S)-2-Methyl-6-undecylpiperidine hydrochloride, (+)-isosolenopsin A hydrochloride 3a

To a solution of $28(0.20 \mathrm{~g}, 0.58 \mathrm{mmol})$ in absolute ethanol $(9.5 \mathrm{~mL})$ was added Raney nickel $(4.38 \mathrm{~g})$. The mixture was placed under hydrogen atmosphere and heated to reflux for $5 \mathrm{~h}$. After cooling, the reaction mixture was filtered on Vericel ${ }^{\circledR}$ membrane and washed with diethylether. The filtrate was concentrated and the crude product was diluted in diethylether $(10 \mathrm{~mL})$. To this solution was added a solution of hydrochloric acid $1 \mathrm{M}$ in diethyl ether $(1 \mathrm{~mL})$. A solid precipitated and was then recrystallized in a mixture of ethanol and AcOEt (1:3). $0.07 \mathrm{~g}$ of $(+)$-isosolenopsin A hydrochloride was obtained. White needles; yield: $43 \% ;[\alpha]_{\mathrm{D}}^{25}=+5.5(c 1.00$, $\left.\mathrm{CHCl}_{3}\right)\left\{\right.$ lit. $\left.{ }^{14 \mathrm{c}}[\alpha]_{\mathrm{D}}^{25}=+10\left(c 1.17, \mathrm{CHCl}_{3}\right)\right\}$; ee $=64 \%$; $\mathrm{mp}=135-140{ }^{\circ} \mathrm{C}$ (lit. ${ }^{14 \mathrm{c}} \mathrm{mp}=150-151^{\circ} \mathrm{C}$ ); spectral data are identical to literature; ${ }^{14 \mathrm{~b}}$ MS (ESI) $\mathrm{m} / \mathrm{z}$ for $\mathrm{C}_{17} \mathrm{H}_{35} \mathrm{NCl}-\mathrm{HCl}+\mathrm{H}: 253\left(\mathrm{M}+\mathrm{H}-\mathrm{HCl}^{+}\right)$.

\section{References}

1. Struntz, G. M.; Findlay, J. A. In The Alkaloids; Brossi, A., Ed.; Academic: New York, 1985; Vol. 26, pp 89-193.

2. Attygalle, A. B.; Xu, S. C.; Mc Cormick, K. D.; Meinwald, J.; Blankespoor, C. L.; Eisner, T. Tetrahedron 1993, 49, 9333-9342.

3. Leclerq, S.; Thirionet, I.; Broeders, F.; Daloze, D.; Van der Meer, R.; Braeckman, J. C. Tetrahedron 1994, 50, 8465-8478.

4. Edwards, M. W.; Daly, J. W. J. Nat. Prod. 1988, 51, 11881197.

5. For recent reviews on the synthesis of piperidines: (a) Bailey, P. D.; Millwood, P. A.; Smith, P. D. J. Chem. Soc., Chem. Commun. 1998, 633-640; (b) Laschat, S.; Dickner, T. Synthesis 2000, 13, 1781-1813; (c) Weintraub, P. M.; Sabol, J. S.; Kane, J. M.; Borcherding, D. R. Tetrahedron 2003, 59, 2953-2989; (d) Buffat, M. G. P. Tetrahedron 2004, 60, 1701-1729.
6. For recent reviews on the stereoselective synthesis of 2,6dialkylpiperidines, see: (a) Husson, H.-P.; Royer, J. Chem. Soc. Rev. 1999, 28, 383-394; (b) Davis, F. A.; Chao, B.; Fang, T.; Szenczyck, J. M. Org. Lett. 2000, 2, 1041-1043; (c) Agami, C.; Couty, F.; Mathieu, H. Tetrahedron Lett. 1998, 39, 3505-3508; (d) Felpin, F. X.; Lebreton, J. Eur. J. Org. Chem. 2003, 3693-3712; (e) Molander, G. A.; Dowdi, E. D.; Pack, S. K. J. Org. Chem. 2001, 66, 4344 4347; (f) Kuethe, J. T.; Comins, D. L. Org. Lett. 2000, 2, 855-857; (g) Carbonnel, S.; Troin, Y. Heterocycles 2002, 10, 1807.

7. Schneider, M. In Alkaloids: Chemical and Biological Perspectives; Pelletier, S. W., Ed.; Pergamon: Oxford, 1996; Vol. 10, pp 155-299.

8. (a) Cellier, M.; Gelas-Mialhe, Y.; Husson, H.-P.; Perrin, B.; Remuson, R. Tetrahedron: Asymmetry 2000, 11, 3913 3919; (b) Chalard, P.; Remuson, R.; Gelas-Mialhe, Y.; Gramain, J.-C.; Canet, I. Tetrahedron Lett. 1999, 40, 1661-1664, and references cited therein.

9. Monfray, J.; Gelas-Mialhe, Y.; Gramain, J.-C.; Remuson, R. Tetrahedron Lett. 2003, 44, 5785-5787.

10. (a) Daub, G. W.; Heerding, D. A.; Overman, L. E. Tetrahedron 1988, 44, 3919-3930; (b) Wu, X.-D.; Khim, S.-K.; Zhang, X.; Cederstrom, E. M.; Mariano, P. S. J. Org. Chem. 1998, 63, 841-859.

11. Daly, J. W.; Nishizawa, Y.; Edwards, M. W.; Waters, J. A.; Aaronstam, R. S. Neurochem. Res. 1991, 16, 489.

12. Edwards, M. W.; Garrafo, H. M.; Daly, J. W. Synthesis 1994, 1167-1170.

13. (a) Chênevert, R.; Dickman, M. J. Org. Chem. 1996, 61, 3332-3341; (b) Ciblat, S.; Calinaud, P.; Canet, J.-L.; Troin, Y. J. Chem. Soc., Perkin Trans. 1 2000, 353-357; (c) Ma, D.; Sun, H. Org. Lett. 2000, 2, 2503-2505; (d) Davis, F. A.; Chao, B.; Rao, A. Org. Lett. 2001, 3, 31693171.

14. For previous stereoselective synthesis of isosolenopsin A: (a) Jefford, C. W.; Wang, J. B. Tetrahedron Lett. 1993, 34, 2911-2914; (b) Poerwono, H.; Higashiyama, K.; Yamauchi, T.; Kubo, H.; Ohmiya, S.; Takahashi, H. Tetrahedron 1998, 54, 13955-13970; (c) Ciblat, S.; Besse, P.; Papastergiou, V.; Veschambre, H.; Canet, J.-L.; Troin, Y. Tetrahedron: Asymmetry 2000, 11, 2221-2229. 